Article

\title{
Synthesis and Spectral Study of a New Family of 2,5-Diaryltriazoles Having Restricted Rotation of the 5-Aryl Substituent
}

\author{
Biligma Tsyrenova and Valentine Nenajdenko * \\ Department of Chemistry, Lomonosov Moscow State University, 119899 Moscow, Russia; tbiligma@gmail.com \\ * Correspondence: nenajdenko@org.chem.msu.ru or nenajdenko@gmail.com; Tel./Fax: +7(495)-939-22-76
}

Academic Editor: Carla Boga

Received: 17 December 2019; Accepted: 22 January 2020; Published: 23 January 2020

check for updates

\begin{abstract}
Efficient synthesis of 2,5-diaryl substituted 4-azido-1,2,3-triazoles by the reaction of sodium azide with dichlorosubstituted diazadienes was demonstrated. The optical properties of the prepared azidotriazoles were studied to reveal a luminescence maximum in the 360-420 nm region. To improve the luminescence quantum yields a family of 4-azido-1,2,3-triazoles bearing ortho-propargyloxy substituents in the 5 position was prepared. Subsequent intramolecular thermal cyclization permits to construct additional triazole fragment and obtain unique benzoxazocine derivatives condensed with two triazole rings. This new family of condensed heterocycles has a flattened heterocyclic system structure to provide more conjugation of the 5-aryl fragment with the triazole core. As a result, a new type of UV/“blue light-emitting” materials with better photophysical properties was obtained.
\end{abstract}

Keywords: triazole; azide; synthesis; cyclization; fluorescence; stokes shift; quantum yield; solvatochromism

\section{Introduction}

1,2,3-Triazoles are an important class of heterocyclic compounds of both theoretical and practical interest [1-4]. This type of heterocycles has been known for more than 150 years, however, during the few last decades, 1,2,3-triazoles have become an attractive object of research in heterocyclic chemistry due to their highly efficient synthesis through copper(I)-catalyzed acetylene-azide cycloaddition [5-13]. A large number of heterocycles containing an embedded triazole core have attracted considerable attention because of their broad range of pronounced biological activity. For example, 1,2,3-triazole derivatives exhibit anti-inflammatory, anesthetic, antimicrobial, anti-arrhythmic, antitumor, and antiviral properties [14-17]. In addition, 1,2,3-triazole derivatives are used in agrochemistry as insecticides, fungicides and plant growth regulators [18].

Nowadays, there is also significant interest in the development of novel selective approaches to 2-substituted 1,2,3-triazoles. These compounds are particularly attractive since they have excellent fluorophore properties [19-25]. A great number of fluorophores has been developed over the years, but effective UV/blue-light-emitting molecules are still rare due to their relatively high energy gap, which may cause poor photostability or low quantum efficiency. However, the booming research area, such as OLED display study, in photoactive compounds has generated increasing needs for effective UV/blue-light-emitting molecules. Recently Yan and co-workers [22] reported N-2-aryl-1,2,3-triazoles (Figure 1) as new UV/blue-light-emitting compounds with tunable emission and adjustable Stokes shift through planar intramolecular charge transfer. 


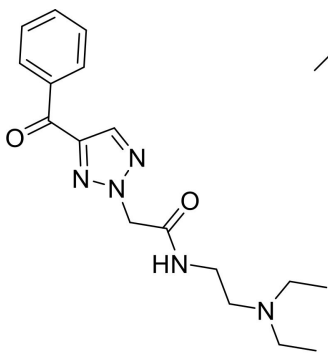

anesthethic

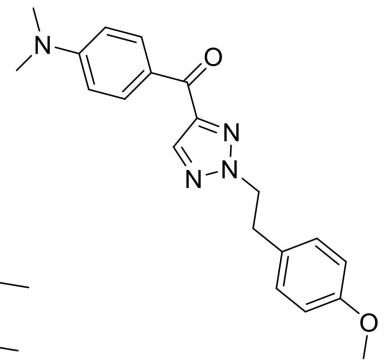

antiarrhythmic agent

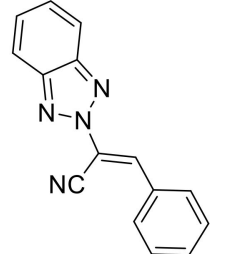

anti-tuberculosis drug

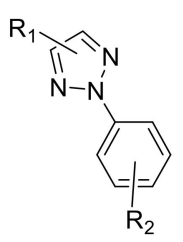

N-2-aryl-1,2,3-triazoles

Figure 1. 2-Substituted 1,2,3-triazoles in medicine and generalized structure of UV/blue-light-emitting $\mathrm{N}-2$-aryl-1,2,3-triazoles.

1,1-Dichlorodiazadienes are a valuable class of electrophiles. These compounds can be prepared using the reaction of carbon tetrachloride witH-N-substituted hydrazones of aldehydes in the presence of $\mathrm{CuCl}$ as a catalyst. [26,27] Recently we demonstrated that these compounds are also interesting diazodyes [28,29]. The reaction of 4,4-dichloro-1,2-diazabuta-1,3-dienes with sodium azide has been found to open straightforward access to extremely rare 1,1-bis-azides (Figure 2). These highly unstable compounds are prone to eliminate a $\mathrm{N}_{2}$ molecule to cyclize into 4-azido-1,2,3-triazoles $\mathbf{1}$ bearing two aryl (heteroaryl) groups at positions 2 and 5 . The reaction was found to be very general for the highly efficient synthesis of various 4-azidotriazoles. It was demonstrated that these heterocycles are highly attractive building blocks for subsequent preparation of 1,2,3-triazole- derived compounds [30].

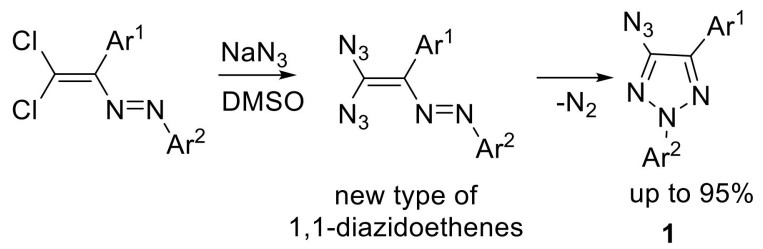

Figure 2. The formation of 4-azido-1,2,3-triazoles.

The prepared azidotriazoles 1 [30] contain the same structural pattern as the diaryltriazoles used as UV/blue-light-emitting compounds [22] (Figure 1). Therefore, we decided to study optical properties of 4-azido-1,2,3-triazoles 1 . This study is also devoted to investigation of the synthesis of 4-azido-2,5-diaryl-1,2,3-azidotriazoles prepared from arylhydrazines and ortho-propargyloxy-benzaldehydes. The presence in the structure of these compounds of a triple bond and the azido group opens up the possibility of intramolecular cyclization of these compounds to obtain unique condensed heterocycles containing two triazole rings (Figure 3b). Moreover, such structural modification can significantly affect the photophysical properties of the intramolecular cyclization products. In this case free rotation of the aryl fragment in the 5 position is impossible. The main aim of this work was studying of optical properties of 2-aryl 1,2,3-trizoles 1 and synthesis of their rigidified analogues obtained via intramolecular acetylene-azide cyclization (Figure $3 b$ ). 

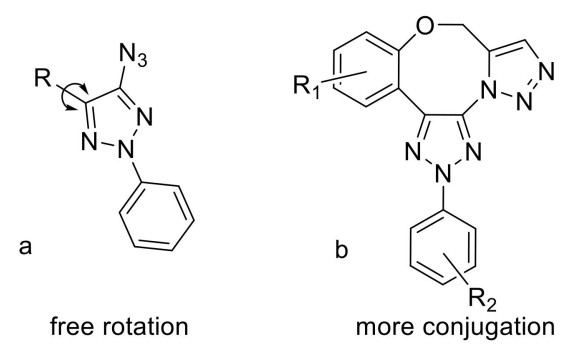

Figure 3. Structures of studied heterocycles. (a) 4-azido-1,2,3-triazoles having free rotation of substituent in the position 5 . (b) rigidified derivatives having restricted rotation.

\section{Results and Discussion}

\subsection{Optical Properties of 4-Azidotriazoles 1}

First, the photophysical data were obtained for family of 2-phenyl-5-aryl-4-azido-1,2,3-triazoles 1a-i [30] having free rotation of the aryl(hetaryl) fragment in the 5 position of the triazole core (Figure 3a, Table 1). Compounds $\mathbf{1 b} \mathbf{b} \mathbf{- 1} \mathbf{i}$ exhibited bathochromic shifts in their absorption spectra relative to $\mathbf{1 a}$ and showed strong maxima in the 311-346 nm region (Figure 4). The strongest bathochromic shift was observed for $\mathbf{1 b}$ containing an electron-withdrawing $\mathrm{NO}_{2}$-group.

The emission spectra of compounds 1a-i have two trends: spectra $\mathbf{1 a}-\mathbf{1 d}$ and $\mathbf{1 g}-\mathbf{1 i}$ exhibited similar emission bands in the 363-372 nm range. On the contrary, the emission spectra of pyridine analogues $1 \mathbf{e}$ and $\mathbf{1 f}$ demonstrated slightly different character having maxima at $419 \mathrm{~nm}$ and Stokes shifts up to $108 \mathrm{~nm}$ (Figure 5, Table 1). The reasons of such behavior are not clear at the moment and demand subsequent study, however it is most probably connected with the presence of additional nitrogen in the structure. Unfortunately, small fluorescence quantum yields were observed for all these compounds 1a-1i (Table 1).

Table 1. Photophysical data of compounds 1a-1i.

\begin{tabular}{|c|c|c|c|c|c|c|}
\hline № & $\mathbf{R}$ & $\lambda_{\mathrm{abs}}, \mathrm{nm}$ & $\lambda_{\mathrm{em}}, \mathrm{nm} *$ & $\lg \varepsilon$ & $\Phi_{\mathrm{F}}^{* *}$ & Stokes Shift, nm \\
\hline $1 \mathbf{a}$ & $\mathbf{P h}$ & 264 & 347 & 3.99 & 0.011 & 83 \\
\hline $1 b$ & $4-\mathrm{NO}_{2}-\mathrm{C}_{6} \mathrm{H}_{4}$ & 346 & 363 & 4.31 & 0.009 & 17 \\
\hline 1c & $4-\mathrm{NMe}_{2}-\mathrm{C}_{6} \mathrm{H}_{4}$ & 321 & 372 & 4.50 & 0.017 & 51 \\
\hline $1 d$ & $4-\mathrm{MeO}-\mathrm{C}_{6} \mathrm{H}_{4}$ & 321 & 370 & 4.23 & 0.020 & 49 \\
\hline $1 e$ & 2-Py & 311 & 419 & 4.34 & 0.013 & 108 \\
\hline 1f & 3-Py & 311 & 419 & 4.53 & 0.015 & 108 \\
\hline $1 \mathrm{~g}$ & 4-Py & 315 & 393 & 4.40 & 0.003 & 78 \\
\hline 1h & 2-quinolyl & 325 & 370 & 4.53 & 0.017 & 45 \\
\hline $1 \mathrm{i}$ & 3-thiophenyl & 316 & 371 & 4.41 & 0.014 & 55 \\
\hline
\end{tabular}




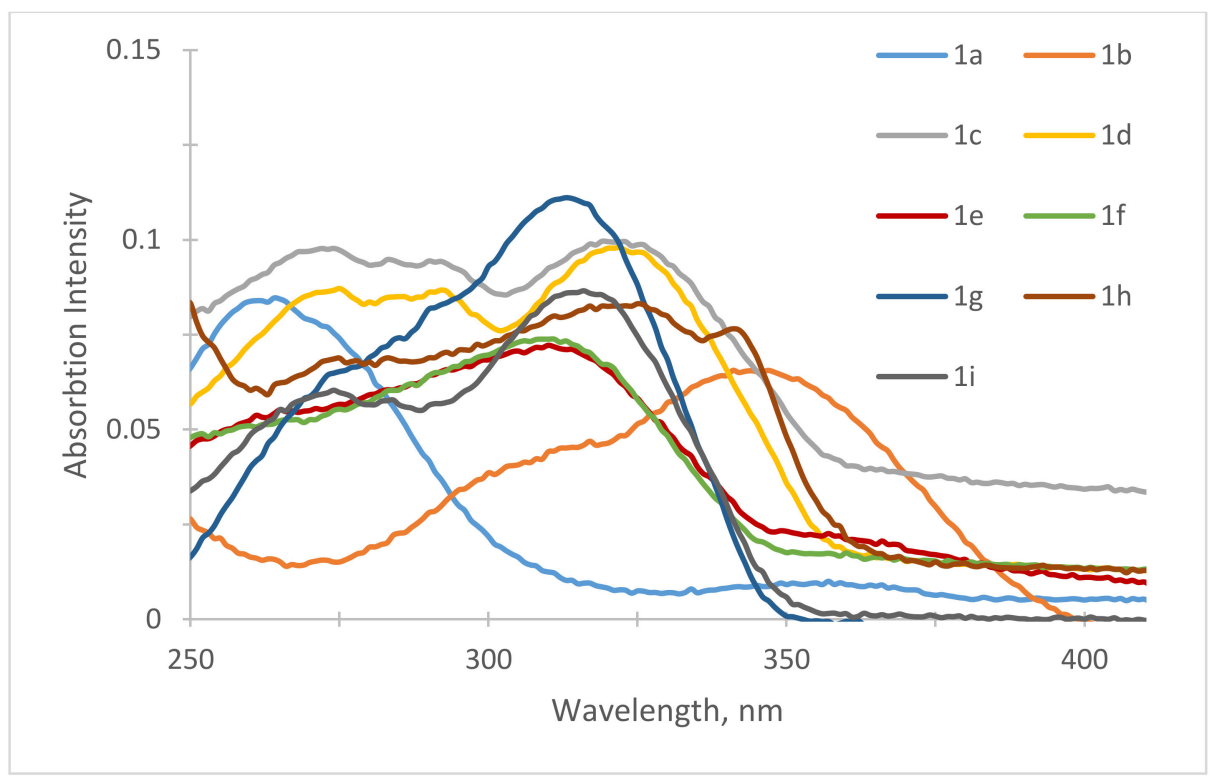

Figure 4. Absorption spectra of $\mathbf{1 a - 1 i}$ in dichloromethane at room temperature.

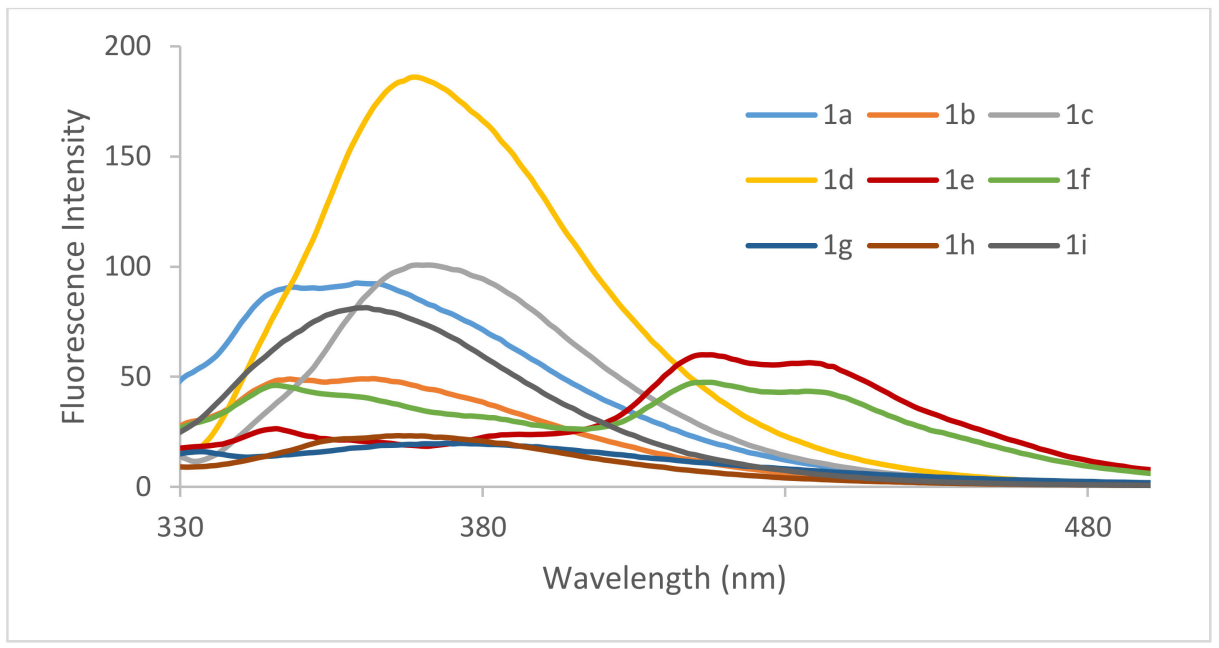

Figure 5. Fluorescence spectra of $\mathbf{1 a - 1 i}$ in dichloromethane at room temperature.

\subsection{Synthesis and Characterization of Condensed Analogues}

Next, we decided to prepare some analogues of compounds 1a-1i having a flattened structure and restricted rotation of the aryl fragment at the 5vposition of the triazole. We expected to enhance the fluorescence quantum yields by such a structural transformation. ortho-Propagyloxy- benzaldehydes were used as starting materials for this aim. A set of such aldehydes was prepared by alkylation of some salic aldehydes with propargyl bromide (Scheme 1).

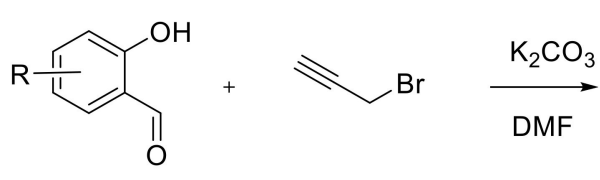

$\mathrm{R}: \mathrm{H}, 3-\mathrm{MeO}, 3-\mathrm{NO}_{2}, 5-\mathrm{NO}_{2}, 5-\mathrm{Br}, 3,5-2 \mathrm{t}-\mathrm{Bu}$, naphtyl<smiles>[R][R]1ccc(OCC#C)c(C=O)c1</smiles>

up to $90 \%$

Scheme 1. Synthesis of ortho-propargyloxybenzaldehydes [31]. 
The prepared o-propargyloxybenzaldehydes were converted into the corresponding 4,4-dichloro-1,2-diaza-1,3-dienes using the one-pot procedure previously elaborated by our team. This modification of the synthesis of 4,4-dichloro-1,2-diaza-1,3-dienes permits one to avoid isolation of hydrazones (See SI).

All steps of the synthesis were performed in one pot to give the target dienes in respectable yields (up to $77 \%$ ). It should be pointed out that the method is amenable to the variation of functional groups in the structure of the starting aldehydes. Electronically and sterically different $o$-propargyloxybenzaldehydes can be used for this aim. Moreover, the corresponding naphthalene derivative was prepared as well (Schemes 2 and 3) [26]. In a similar manner, a set of dienes $\mathbf{2 h}-\mathbf{h}$ having different aryl substituents at the nitrogen was prepared from the parent o-propargyloxy-benzaldehyde and substituted aryl hydrazines. We tried to perform variation of this part of molecules keeping in mind the influence of both electron and steric factors. For example, the corresponding dienes having methyl-, methoxy- and cyano groups, and different halogens can be prepared in up to $89 \%$ yield. Moreover, diene $\mathbf{2 m}$ having a bulky 2,6-dimethylphenyl substituent was synthesized too.

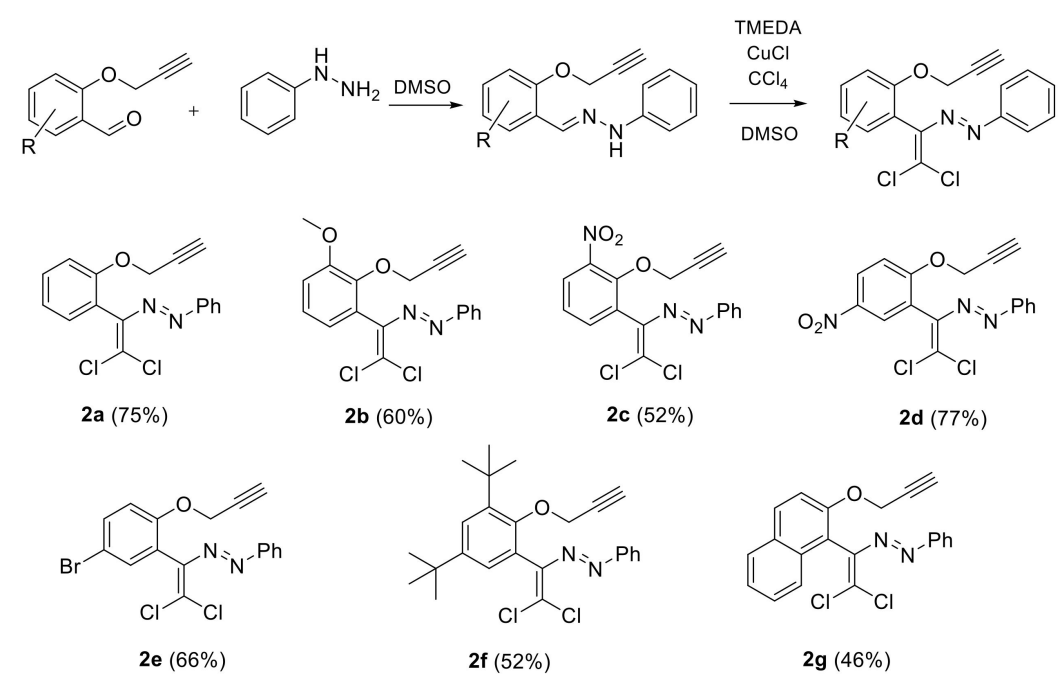

Scheme 2. Synthesis of $o$-propargyloxy-substituted dichlorodiazadienes (showing the possible diversity of the aldehyde moiety).

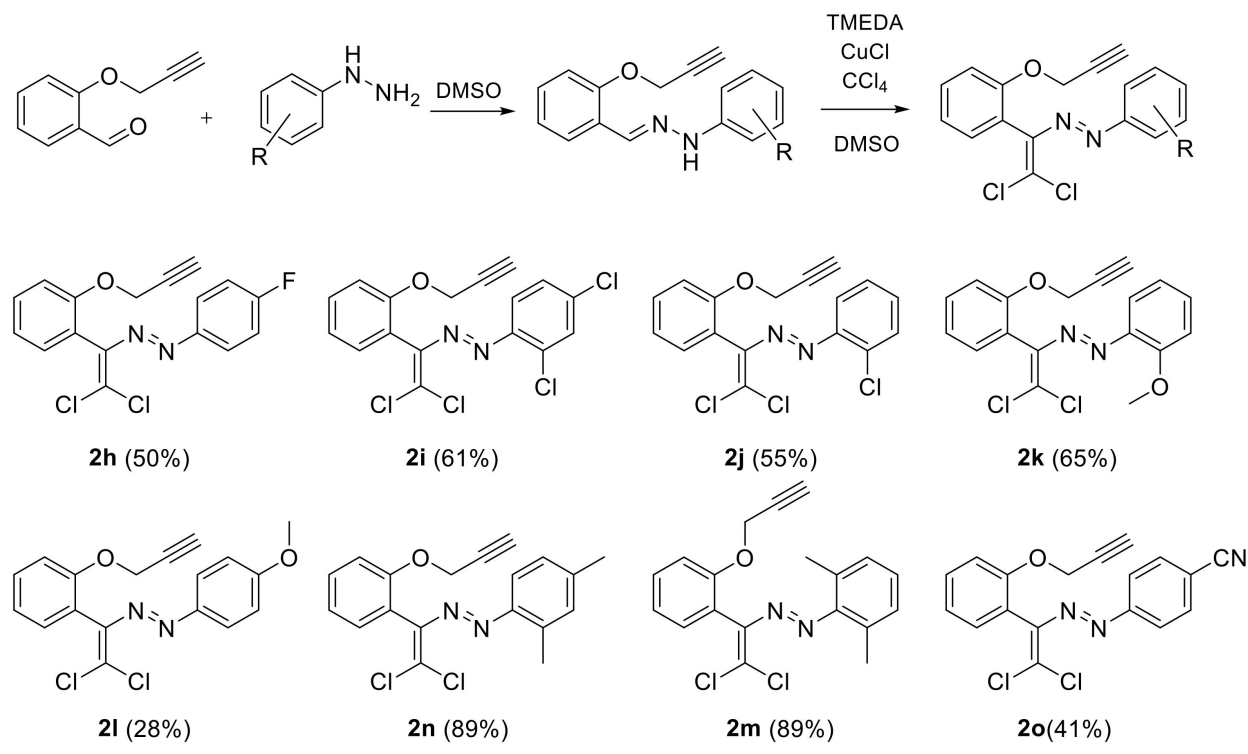

Scheme 3. Synthesis of o-propargyloxy-substituted dichlorodiazadienes (showing the possible diversity of the hydrazine moiety). 
Having in hand a family of precursors for the synthesis of model azidotriazoles, the reaction with excess of sodium azide was studied. It was found that the synthesis is very general to give the target products in up to $97 \%$ yield. A set of 15 triazoles $3 \mathbf{a}-\mathbf{0}$ was thus prepared having different substituents in the position 2 and 5 (Scheme 4) [30,32,33].

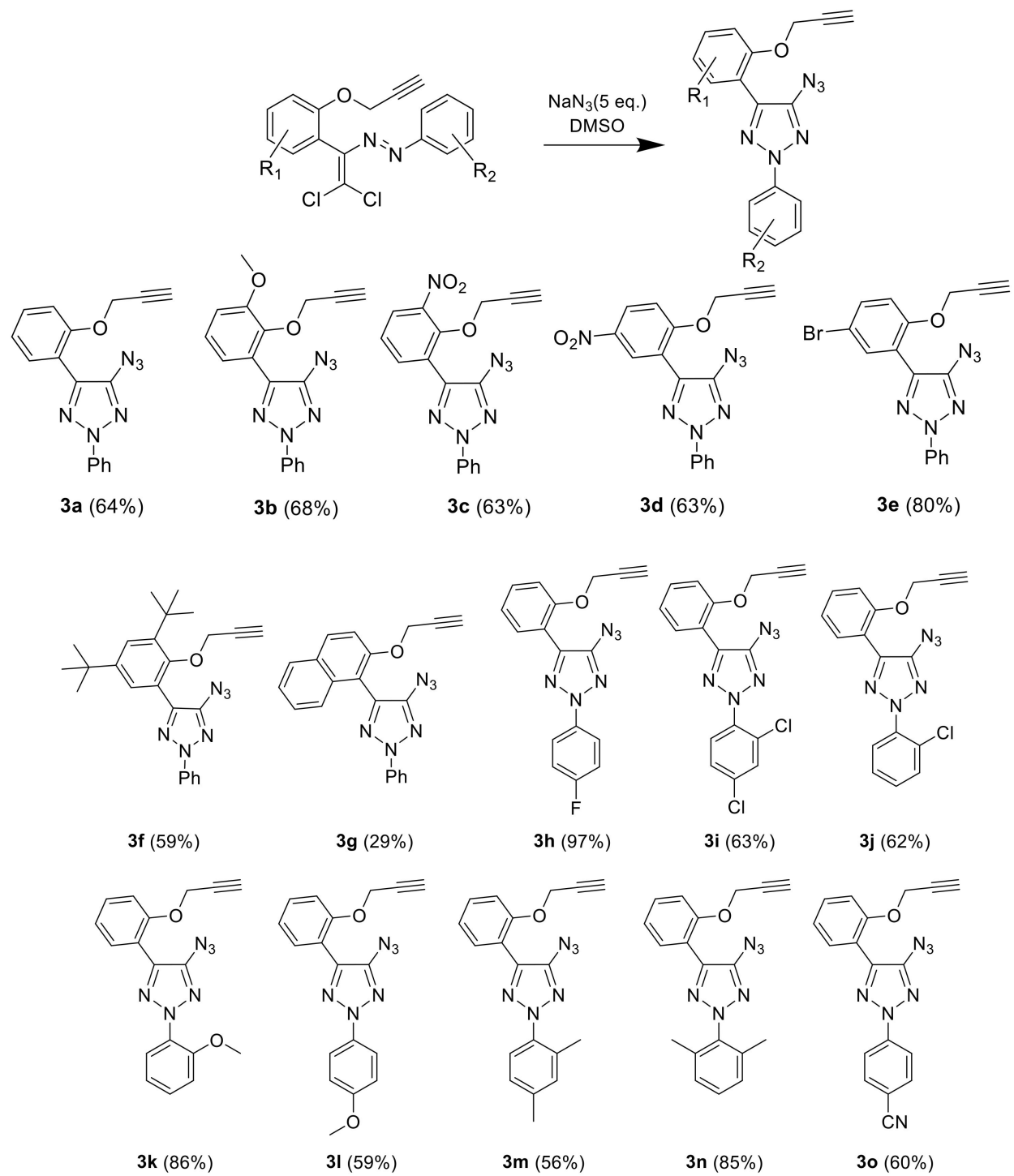

Scheme 4. Synthesis of o-propargyloxy-substituted 4-azido-2,5-diaryl-1,2,3-triazoles.

Next, intramolecular cyclization to form second triazole ring by thermal [2+3] cycloaddition was studied. Prepared compounds 3 have in the structure both an azide group and an acetylene fragment. We observed that spontaneous cyclization takes place slowly, even at room temperature, during storage. Smooth cyclization can be performed by reflux in o-xylene during $12 \mathrm{~h}$ in an argon atmosphere. As a result, a family of condensed triazole derivatives 4 having restricted rotation of substituent at the 5 position was prepared. We observed atropoisomerism [34] for some of prepared products. Their NMR spectra contain doubled set of signals (Scheme 5). 


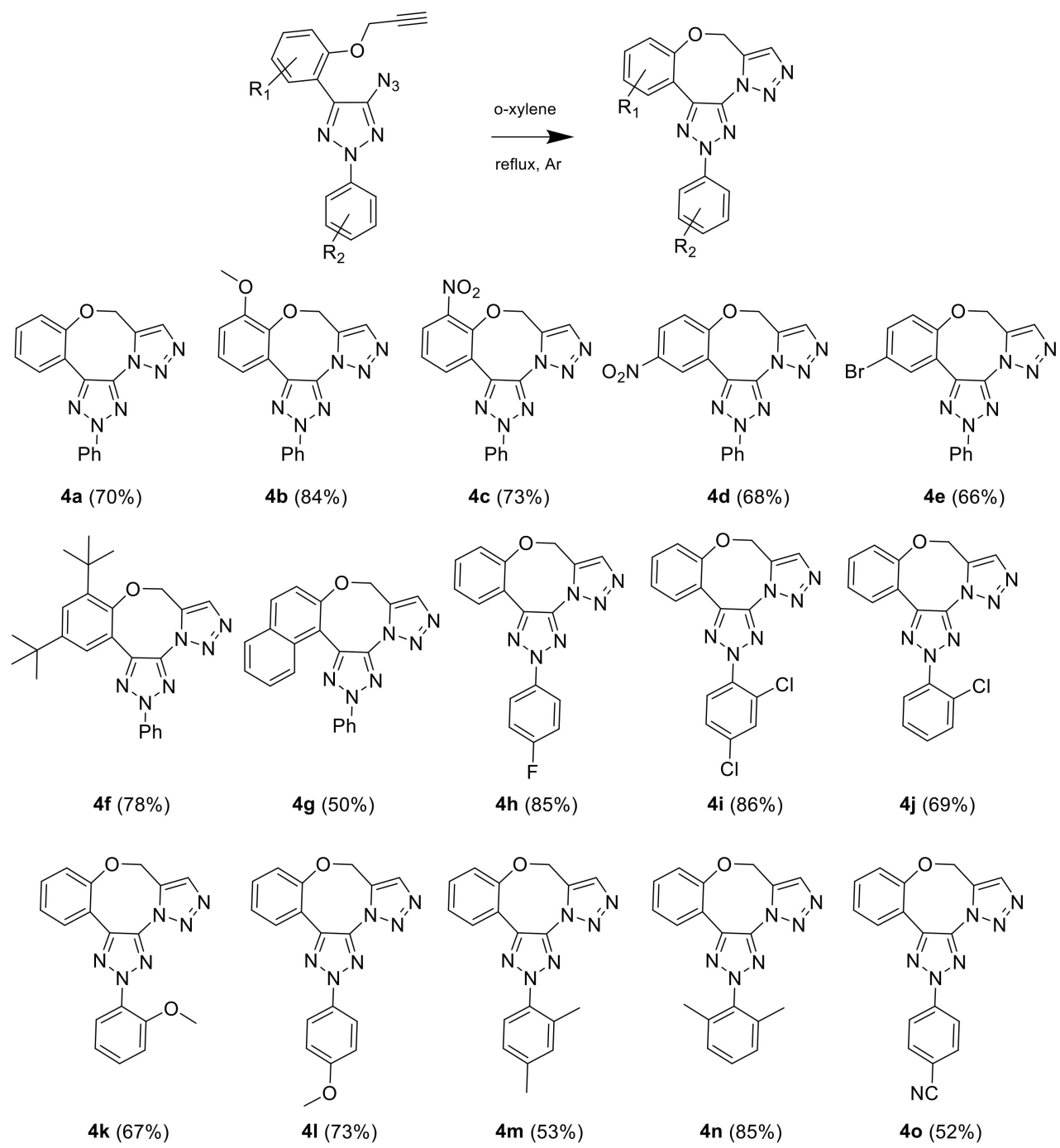

Scheme 5. Intramolecular cyclization of o-propargyloxy substituted 4-azido-2,5-diaryl-1,2,3-triazoles.

\subsection{Photophysical Properties of Compounds $\mathbf{4 a}-\mathbf{4 o}$}

UV-vis absorption and fluorescence spectroscopic measurements were performed for the synthesized compounds 4 to establish the relationship between the structure and photophysical properties of the prepared flattened derivatives $4 \mathbf{4}-\mathbf{o}$ [35]. All these spectral data were obtained in dichloromethane ( $\mathrm{c}=10^{-6} \mathrm{M}$ for all compounds) at room temperature and the results are summarized in Table 2.

All the investigated compounds exhibited similar absorptions in the 250-305 $\mathrm{nm}$ range (Figure 6). The absorption spectra for compounds having a phenyl group at the $\mathrm{N}(2)$ position demonstrated absorption maxima at $284-288 \mathrm{~nm}$. However, naphthalene derivative $4 \mathrm{~g}$ has a maximum of absorption shifted to $301 \mathrm{~nm}$. More pronounced influence for absorption spectra was found when varying the substituents at $\mathrm{N}(2)$. The presence of an electron-withdrawing cyano group resulted in a bathochromic shift to $305 \mathrm{~nm}$. On the other hand, a hypsochromic shift was observed for ortho-substituted derivatives, for example $4 \mathbf{k}$. 
Table 2. Photophysical data of compounds $4 \mathbf{a}-\mathbf{4 o}$ in solution $\left(\mathrm{CH}_{2} \mathrm{Cl}_{2}\right)$.

\begin{tabular}{|c|c|c|c|c|c|c|c|}
\hline № & $\mathbf{R}_{\mathbf{1}}$ & $\mathbf{R}_{2}$ & $\lambda_{\mathrm{abs},}, \mathrm{nm}$ & $\lambda_{\mathrm{em}}, \mathrm{nm} *$ & $\lg \varepsilon$ & $\Phi_{\mathrm{F}} * *$ & Stokes Shift, nm \\
\hline $4 a$ & $\mathbf{H}$ & $\mathbf{H}$ & 288 & 353 & 4.49 & 0.044 & 65 \\
\hline $4 b$ & 3-MeO & $\mathbf{H}$ & 286 & 342 & 4.51 & 0.018 & 56 \\
\hline $4 c$ & $3-\mathrm{NO}_{2}$ & $\mathbf{H}$ & 284 & 317 & 4.61 & 0,009 & 33 \\
\hline $4 d$ & $5-\mathrm{NO}_{2}$ & $\mathbf{H}$ & 287 & 318 & 4.44 & 0,007 & 31 \\
\hline $4 e$ & $5-\mathrm{Br}$ & $\mathbf{H}$ & 287 & 355 & 4.13 & 0.032 & 68 \\
\hline $4 f$ & 3,5-di-t-Bu & $\mathbf{H}$ & 287 & 329 & 4.45 & 0.009 & 42 \\
\hline $4 g$ & Naphthyl & $\mathbf{H}$ & 301 & 356 & 4.39 & 0.079 & 55 \\
\hline $4 h$ & $\mathbf{H}$ & $4-\mathrm{F}$ & 287 & 352 & 4.44 & 0.042 & 65 \\
\hline $4 i$ & $\mathbf{H}$ & $2,4-2 \mathrm{Cl}$ & 274 & 357 & 4.18 & 0.040 & 83 \\
\hline $4 j$ & $\mathbf{H}$ & $2-\mathrm{Cl}$ & 259 & 354 & 4.46 & 0.001 & 95 \\
\hline $4 k$ & $\mathbf{H}$ & 2-MeO & 255 & 367 & 4.35 & 0.013 & 112 \\
\hline 41 & $\mathbf{H}$ & 4-MeO & 299 & 376 & 4.54 & 0.616 & 77 \\
\hline $4 m$ & $\mathbf{H}$ & 2,4-2Me & 267 & 357 & 4.53 & 0.002 & 90 \\
\hline $4 n$ & $\mathbf{H}$ & $2,6-2 \mathrm{Me}$ & 250 & 317 & 4.46 & 0.003 & 67 \\
\hline 40 & $\mathbf{H}$ & $4-\mathrm{CN}$ & 305 & 372 & 4.51 & 0.198 & 67 \\
\hline
\end{tabular}

${ }^{*} \mathrm{UV}$-Vis and fluorescence spectra are measured at room temperature and concentration $10^{-6} \mathrm{M}$. ${ }^{* *}$ Fluorescence quantum yields are measured relative to $0.1 \mathrm{M} \mathrm{H}_{2} \mathrm{SO}_{4}$ solution of quinine $\left(\Phi_{\mathrm{f}}=0.55\right)$ and $0.1 \mathrm{M} \mathrm{H}_{2} \mathrm{SO}_{4}$ solution of 2-aminopyridine $\left(\Phi_{\mathrm{f}}=0.60\right)$.

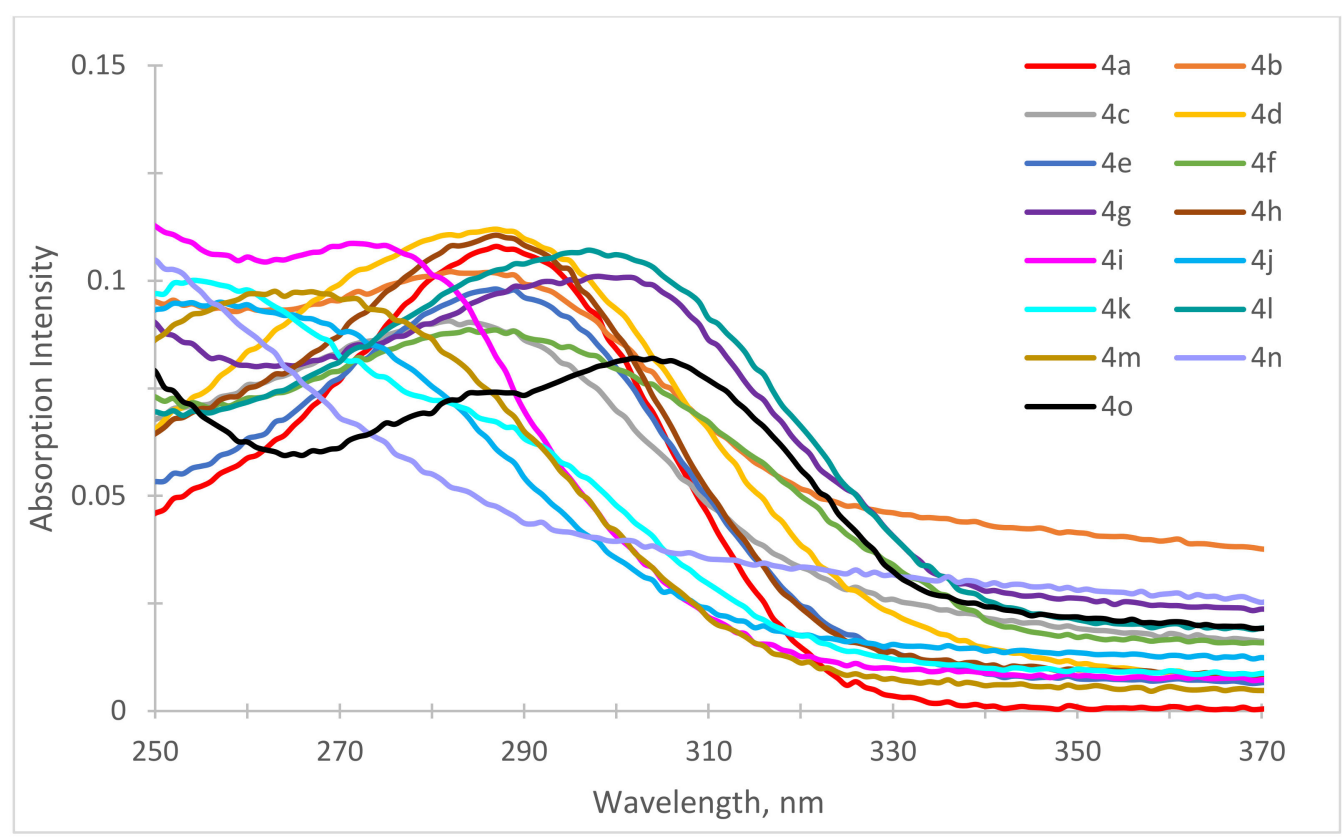

Figure 6. Absorption spectra of $\mathbf{4 a - 4 o}$ in dichloromethane at room temperature.

The emission spectra of solutions of 4a-4o (Figure 7) were recorded at an excitation wavelength corresponding to the maximum in the absorption spectra. Typical emission maxima obtained upon irradiation of the solutions were located in the blue region. The Stokes shifts were shown in the range from 31 to $112 \mathrm{~nm}$. 


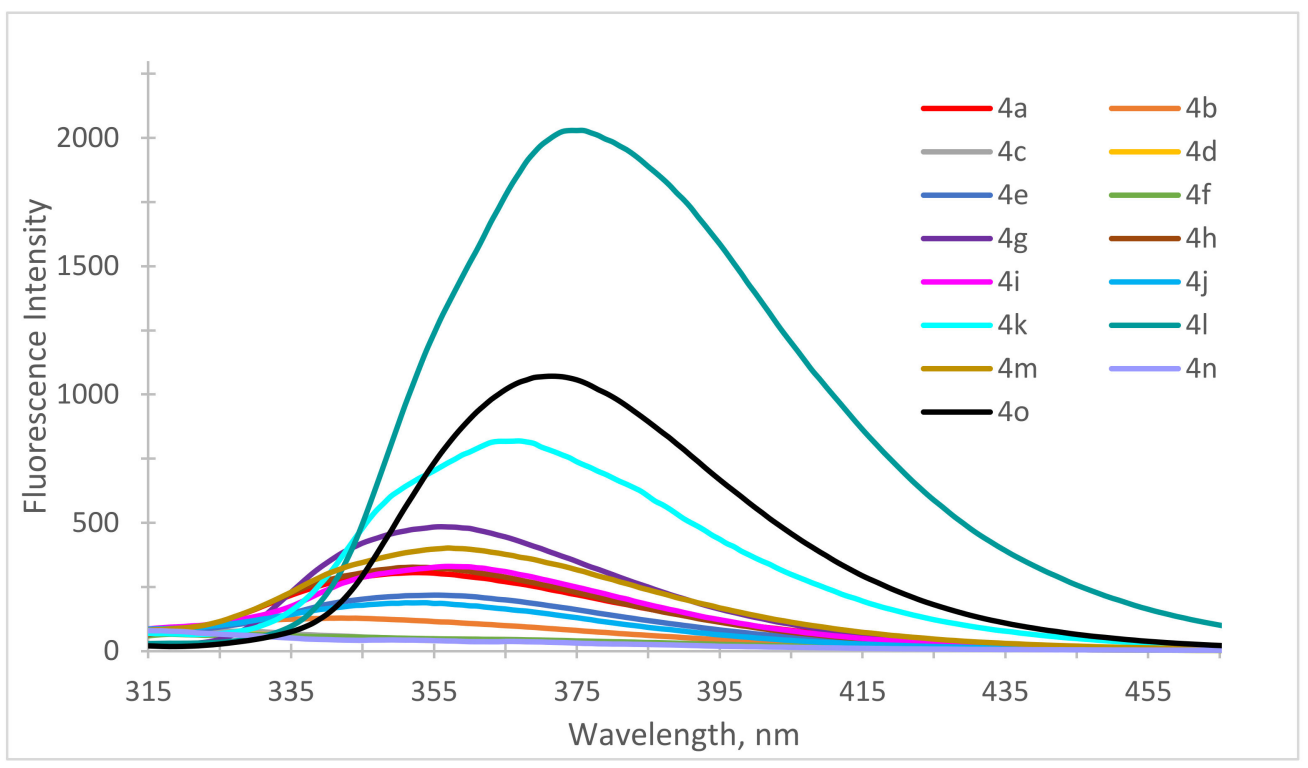

Figure 7. Fluorescence spectra of $\mathbf{4 a - 4 o}$ in dichloromethane at room temperature.

Obviously, the spectral characteristics of compounds $\mathbf{4 a - 4 o}$ depend on their electronic properties, conjugation of the substituents at the $\mathrm{C}(5)$ position and at the $\mathrm{N}(2)$ of the triazole. To our delight, much higher quantum yields ( $\Phi_{\mathrm{F}}$ up to 0.616 ) were observed for all derivatives $4 \mathbf{a}-\mathbf{4 o}$ in comparison to triazole derivatives $\mathbf{1 a}-\mathbf{h}\left(\Phi_{\mathrm{F}}\right.$ up to 0.017$)$ having free rotation of the $C(5)$ substituents. These data confirmed the attractiveness of our idea to synthesize and to study the photophysical properties of flattened intramolecular cyclizatioproducts. Analysis of the emission spectra for compounds $\mathbf{4 a - 4 o}$ showed that the substitution at the $\mathrm{N}(2)$ position has more influence on fluorescence properties. A presence of a methoxy or cyano group at the aromatic ring para-position at the triazole $\mathrm{N}(2)$ leads to enhanced quantum yields (Table 2, Figures 6 and 7), whereas, ortho-substituted derivatives exhibited lower fluorescenc efficiency. In contrast, the quantum yields were below $5 \%$ for any substituents at the $C(5)$ of triazole ring (compounds $4 \mathbf{a}-\mathbf{f}, \Phi_{\mathrm{F}}$ up to $\left.0.007-0.044\right)$. Due to the extra ring the naphthyl derivative $4 \mathrm{~g}$ showed moderate photophysical properties $\left(\Phi_{\mathrm{F}}=0.079\right)$.

The presence of a 4-MeO-group in the aryl at the $\mathrm{N}(2)$-triazole resulted in the most effective conjugation, which resulted in a fluorescence enhancement (comp. 4l, $\Phi_{\mathrm{F}}$ up to 0.616 ). Most probably, better internal charge transfer (ICT) from the electron-donor OMe-group to the relatively electronically deficient part of molecule is achieved. On the contrary, the 2-methoxy derivative has the small quantum yield (comp. 4k, $\Phi_{\mathrm{F}}$ up to 0.013 ) but it gives the largest Stokes shift $(112 \mathrm{~nm})$, that makes it a pretty interesting fluorophore material. Other ortho-substituted at $\mathrm{N}(2)$-triazole derivatives also demonstrated low quantum yields ( $4 \mathbf{i}, \mathbf{4 j}, \mathbf{4 m}$ and $\mathbf{4 n}$ ) to confirm the influence of steric hindrance on the photophysical properties. Most probably, lower conjugation of the aryl ring at the N(2)-triazole due to a distorted conformation is a reason for the reduced quantum yields in these cases.

\subsection{Solvatochromism of Compound $4 l$}

We also carried out a study of solvatochromic properties for compound 41 which demonstrated the highest quantum yields. The absorption and emission spectra of $4 \mathbf{l}$ were taken at a standard concentration in different solvents of various polarities, including dioxane, benzene, EtOAc, THF, dichloromethane, $\mathrm{EtOH}, \mathrm{DMF}, \mathrm{MeCN}$ [36]. The UV-Vis spectrum of 41 in low polarity benzene has a slight bathochromic shift relative to the spectrum in dichloromethane (Figure 8). Increasing solvent polarity resulted in more significant bathochromic shifts of the emission maxima, indicating an ICT behavior, which is better stabilized in polar solvents, for example in THF or EtOH (Figure 9). The intensity of the emission of compound $\mathbf{4 l}$ is also highly dependent on the solvent polarity. In particular, the quantum yield of $4 \mathbf{l}$ rises with increasing polarity of the solvents (benzene $(0.457)$ 
$<$ dioxane [0.497] $<$ DMF [0.540] < EtOAc [0.583] < THF [0.591] $<\mathrm{CH}_{2} \mathrm{Cl}_{2}[0.616]<\mathrm{MeCN}[0.618]<$ $\mathrm{EtOH}[0.670]$ ) (Table 3). The lowest fluorescence quantum yield was observed in the nonpolar, aprotic solvent benzene because of the charge transfer phenomena [37]. The highest fluorescence quantum yield was observed in a polar, protic solvent, EtOH.

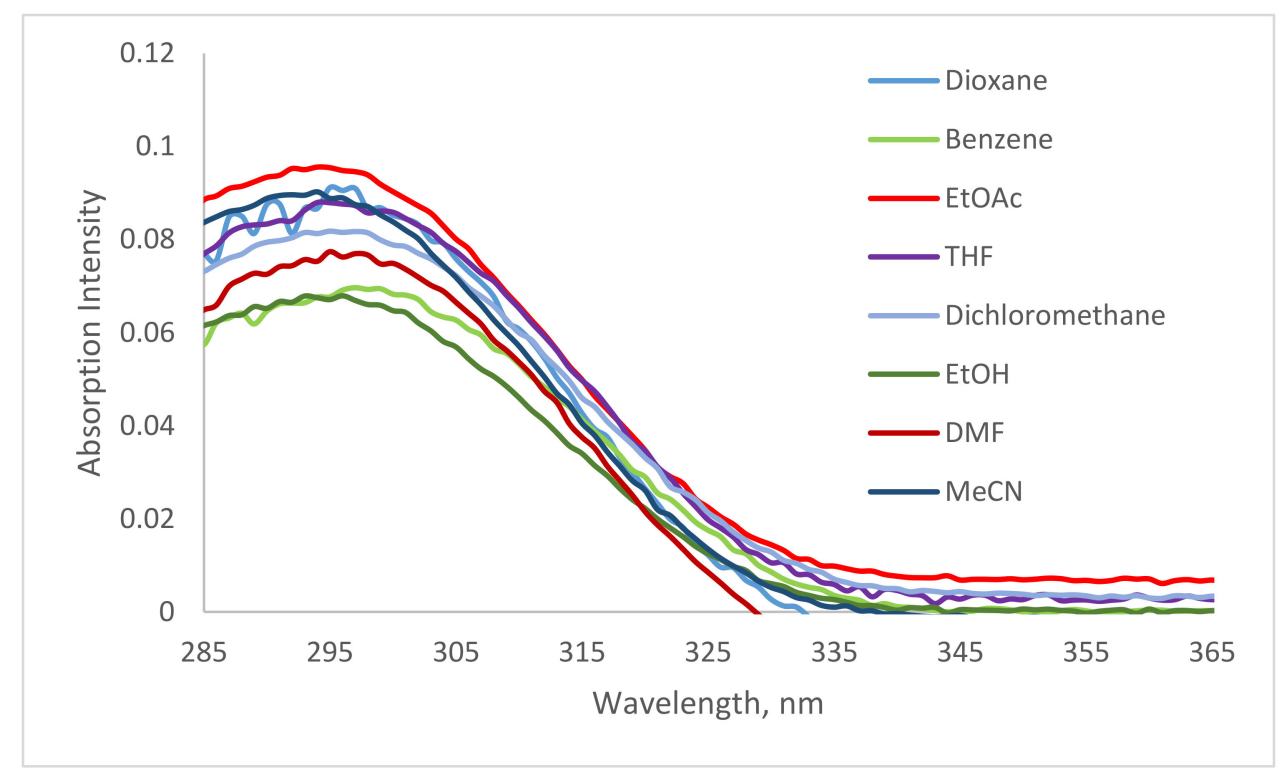

Figure 8. Absorption spectra of 41 in different solvents at room temperature.

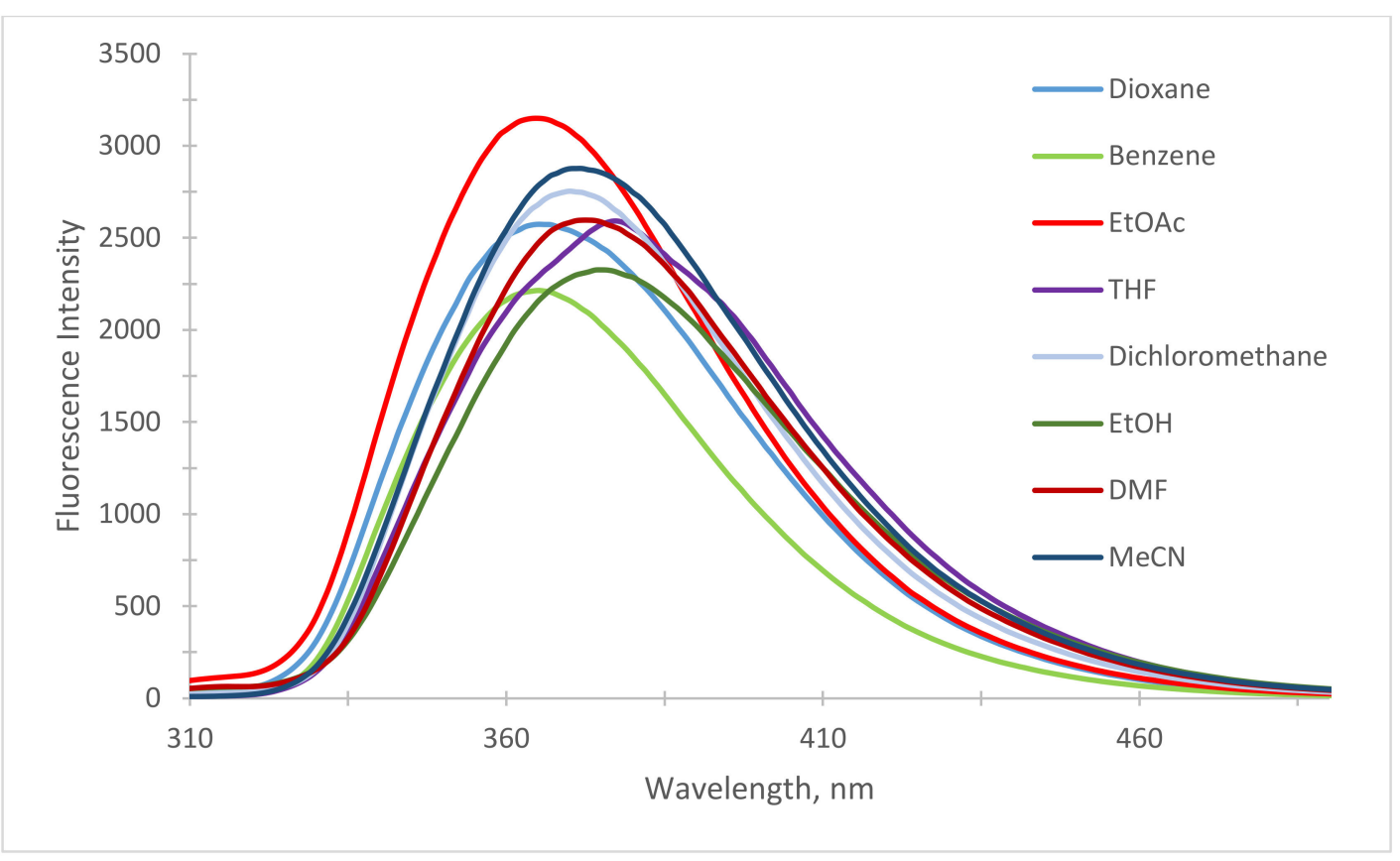

Figure 9. Fluorescence spectra of 41 in different solvents at room temperature. 
Table 3. Quantum yields and Stokes shifts of 41 in different solvents.

\begin{tabular}{cccccc}
\hline Solvent & $\boldsymbol{\lambda}_{\text {abs }}, \mathbf{n m}$ & $\boldsymbol{\lambda}_{\mathbf{e m}}, \mathbf{n m}$ & $\mathbf{l g} \mathcal{E}$ & $\boldsymbol{\Phi}_{\mathbf{F}}$ & Stokes Shift, $\mathbf{n m}$ \\
\hline Dioxane & 296 & 365 & 4.54 & 0.497 & 69 \\
\hline Benzene & 298 & 365 & 4.07 & 0.457 & 67 \\
\hline EtOAc & 294 & 365 & 4.56 & 0.583 & 71 \\
\hline $\mathrm{THF}$ & 295 & 377 & 4.46 & 0.591 & 82 \\
\hline $\mathrm{CH}_{2} \mathrm{Cl}_{2}$ & 296 & 371 & 4.54 & 0.616 & 75 \\
\hline $\mathrm{EtOH}$ & 296 & 379 & 4.36 & 0.670 & 83 \\
\hline $\mathrm{DMF}$ & 297 & 373 & 4.29 & 0.540 & 76 \\
\hline $\mathrm{MeCN}$ & 294 & 372 & 4.19 & 0.618 & 78 \\
\hline
\end{tabular}

\section{Materials and Methods}

\subsection{Experimental Details}

All required fine chemicals were of reagent grade and were used directly without purification unless otherwise noted. ${ }^{1} \mathrm{H}$ - and ${ }^{13} \mathrm{C}-\mathrm{NMR}$ spectra were acquired at 400.1 and $100.6 \mathrm{MHz}$, respectively, on an AVANCE $400 \mathrm{MHz}$ spectrometer (Bruker, Karlsruhe, Germany) in chloroform-d (unless otherwise stated). ${ }^{1} \mathrm{H}-\mathrm{NMR}$ coupling constants $(J)$ are reported in Hertz. Data are reported as follows: chemical shift, multiplicity ( $\mathrm{s}$ - singlet, br s - broad singlet, $\mathrm{d}$ - doublet, $\mathrm{t}$ - triplet, $\mathrm{q}$ - quartet, $\mathrm{m}$ - multiplet, $\mathrm{dd}$ doublet of doublets, ddd - doublet of double doublets), coupling constants, integration, and assignment (optionally). HRMS (ESI-MS) spectra were measured on MicroTof (Bruker Daltonics, Bremen, Germany). All IR data was obtained on a Nicolet iS5 One FT-IR spectrometer (Thermo Scientific, Madison, WI USA) using consoles of internal reflection iS3 with a ZnSe ATR element, dip angle $45^{\circ} \mathrm{C}$. All UV data was obtained on a Cary 60 UV-Visible spectrophotometer (Agilent, Santa Clara, CA USA) within 250-800 nm spectral range. The UV-spectra were recorded at $1 \mathrm{~cm}$ cuvettes at room temperature, dichloromethane was used as a solvent. Emission spectra were registered with a F2700 spectrofluorometer (Hitachi, Tokyo, Japan) in $1 \mathrm{~cm}$ quartz cells. The concentration of the compound in the solutions was $10^{-5} \mathrm{M}$ and $10^{-6} \mathrm{M}$ for both measurements. The relative fluorescence quantum yields $(\Phi \mathrm{F})$ were measured using quinine sulfate in $0.1 \mathrm{M} \mathrm{H} 2 \mathrm{SO} 4\left(\Phi_{\mathrm{f}}=0.55\right)$ and 2-aminopyridine $0.1 \mathrm{M} \mathrm{H} 2 \mathrm{SO} 4\left(\Phi_{\mathrm{f}}=0.60\right)$ as a standards [36].

Analytical TLC: aluminium-backed plates precoated $(0.25 \mathrm{~mm})$ with Silica Gel 60 F254 (Merck, Darmstadt, Germany) and $0.20 \mathrm{~mm}$ ALUGRAM ${ }^{\circledR}$ Xtra SIL G/UV ${ }_{254}$. (Macherey-Nagel, Düren, Germany). Compounds were visualized by exposure to UV light or by dipping the plates in permanganate $\left(\mathrm{KMnO}_{4}\right)$ stain followed by heating. Flash and column chromatography were performed using Macherey-Nagel Silica gel 60 (70-230 mesh). All mixed solvent eluents are reported as v/v solutions. Solvents were purified by standard methods. DMSO was distilled over $\mathrm{CaH}_{2}$. Tetrachloromethane was distilled over $\mathrm{P}_{2} \mathrm{O}_{5}$.

3.2. General Procedure for the Preparation of

(E)-1-(2,2-Dichloro-1-(2-(prop-2-yn-1-yloxy)phenyl)vinyl)-2-Phenyldiazenes

$o$-Propargyloxy-substituted dichlorodiazabutadienes were synthesized based on previous work [26]. A $20 \mathrm{~mL}$ screw neck vial was charged with DMSO $(10 \mathrm{~mL})$, the corresponding o-propargyloxybenzaldehyde [31] ( $1 \mathrm{mmol}, 1$ eq.) and hydrazine (1 eq.). After $2 \mathrm{~h}$ stirring TMEDA (2.5 eq.), $\mathrm{CuCl}(0.01$ eq.) were added and carbon tetrachloride (10 eq.) was put into the reaction mixture during $5 \mathrm{~min}$ under cooling with a water bath. The next reaction was carried out at room temperature during $3 \mathrm{~h}$ (until TLC analysis showed complete consumption of corresponding hydrazone). The reaction mixture was then poured into water $(200 \mathrm{~mL})$, and extracted with DCM $(3 \times 20 \mathrm{~mL})$. The combined organic phase was washed with water $(3 \times 50 \mathrm{~mL})$, brine $(1 \times 30 \mathrm{~mL})$, dried over anhydrous sodium 
sulfate and concentrated in vacuo of the rotary evaporator. The residue was purified by column chromatography on silica gel using appropriate mixtures of hexane and DCM (3/1) as eluent.

(E)-1-(2,2-Dichloro-1-(2-(prop-2-yn-1-yloxy)phenyl)vinyl)-2-phenyldiazene (2a). Yield $248 \mathrm{mg}$ (75\%), orange-red solid, m.p. $85{ }^{\circ} \mathrm{C}$. IR $\left(v, \mathrm{~cm}^{-1}\right): 1573,1584,1604,3261 .{ }^{1} \mathrm{H}-\mathrm{NMR}: \delta 2.45(\mathrm{t}, 1 \mathrm{H}, J=$ $2.4 \mathrm{~Hz}), 4.63(\mathrm{~d}, 2 \mathrm{H}, J=2.4 \mathrm{~Hz}), 7.08-7.15(\mathrm{~m}, 3 \mathrm{H}), 7.42-7.46(\mathrm{~m}, 4 \mathrm{H}), 7.76-7.81(\mathrm{~m}, 2 \mathrm{H}) .{ }^{13} \mathrm{C}-\mathrm{NMR}: \delta$ $56.1,75.5,78.5,112.7,121.3,122.9,123.2,128.9,130.3,131.3,131.3,136.0,149.9,153.0,154.9$. ESI-HRMS $(\mathrm{m} / \mathrm{z})$ : calcd. for $\left(\mathrm{C}_{17} \mathrm{H}_{13} \mathrm{Cl}_{2} \mathrm{~N}_{2} \mathrm{O}\right)\left[\mathrm{M}+\mathrm{H}^{+}\right] 331.0400$, found 331.0400 .

(E)-1-(2,2-Dichloro-1-(3-methoxy-2-(prop-2-yn-1-yloxy)phenyl)vinyl)-2-phenyldiazene (2b). Yield $217 \mathrm{mg}$ $(60 \%)$, orange-red oil. IR $\left(v, \mathrm{~cm}^{-1}\right): 1582,1603,3297 .{ }^{1} \mathrm{H}-\mathrm{NMR}: \delta 2.33(\mathrm{t}, 1 \mathrm{H}, J=2.5 \mathrm{~Hz}), 3.91(\mathrm{~s}, 3 \mathrm{H})$, $4.60(\mathrm{~s}, 2 \mathrm{H}), 6.73(\mathrm{dd}, 1 \mathrm{H}, J=7.7,1.4 \mathrm{~Hz}), 7.01(\mathrm{dd}, 1 \mathrm{H}, J=8.3,1.3 \mathrm{~Hz}), 7.15(\mathrm{t}, 1 \mathrm{H}, J=8.0 \mathrm{~Hz}), 7.42-7.45$ (m, 3H), 7.76-7.80 (m, 2H). ${ }^{13} \mathrm{C}-\mathrm{NMR}: \delta 55.7,60.2,74.9,79.3,113.2,122.5,123.2,124.4,128.0,128.9,131.3$, 136.3, 144.7, 149.9, 152.4, 152.9. ESI-HRMS (m/z): calcd. for $\left(\mathrm{C}_{187} \mathrm{H}_{15} \mathrm{Cl}_{2} \mathrm{~N}_{2} \mathrm{O}_{2}\right)\left[\mathrm{M}+\mathrm{H}^{+}\right] 361.0506$, found 361.0505 .

(E)-1-(2,2-Dichloro-1-(3-nitro-2-(prop-2-yn-1-yloxy)phenyl)vinyl)-2-phenyldiazene (2c). Yield $196 \mathrm{mg}$ (52\%), orange oil. IR $\left(v, \mathrm{~cm}^{-1}\right): 1533,1574,1602,3292 .{ }^{1} \mathrm{H}-\mathrm{NMR}: \delta 2.49(\mathrm{t}, 1 \mathrm{H}, J=2.5 \mathrm{~Hz}), 4.55(\mathrm{~d}, 2 \mathrm{H}, J=9.5$ $\mathrm{Hz}), 7.32-7.37(\mathrm{~m}, 2 \mathrm{H}), 7.43-7.49(\mathrm{~m}, 3 \mathrm{H}), 7.75-7.79(\mathrm{~m}, 2 \mathrm{H}), 7.98(\mathrm{dd}, 1 \mathrm{H}, J=7.0,2.9 \mathrm{~Hz}) .{ }^{13} \mathrm{C}-\mathrm{NMR}: \delta$ 62.6, 76.8, 77.4, 110.5, 123.3, 124.6, 126.3, 129.1, 130.9, 132.0, 133.3, 136.3, 137.6, 144.3, 148.2, 149.5, 152.5 . ESI-HRMS (m/z): calcd. for $\left(\mathrm{C}_{17} \mathrm{H}_{12} \mathrm{Cl}_{2} \mathrm{~N}_{3} \mathrm{O}_{3}\right)\left[\mathrm{M}+\mathrm{H}^{+}\right]$376.0251, found 376.0275.

(E)-1-(2,2-Dichloro-1-(5-nitro-2-(prop-2-yn-1-yloxy)phenyl)vinyl)-2-phenyldiazene (2d). Yield $290 \mathrm{mg}(77 \%)$, orange-red solid, m.p. $105^{\circ} \mathrm{C}$. IR $\left(v, \mathrm{~cm}^{-1}\right)$ : 1568, 1585, 1612, 3290. ${ }^{1} \mathrm{H}-\mathrm{NMR}: \delta 2.53(\mathrm{t}, 1 \mathrm{H}, J=2.4 \mathrm{~Hz})$, $4.74(\mathrm{~d}, 2 \mathrm{H}, J=2.4 \mathrm{~Hz}), 7.20(\mathrm{~d}, 1 \mathrm{H}, J=9.2 \mathrm{~Hz}), 7.41-7.48(\mathrm{~m}, 3 \mathrm{H}), 7.72-7.77(\mathrm{~m}, 2 \mathrm{H}), 8.06(\mathrm{~d}, 1 \mathrm{H}, J=2.8$ $\mathrm{Hz}), 8.35$ (dd, $1 \mathrm{H}, J=9.2,2.8 \mathrm{~Hz}) .{ }^{13} \mathrm{C}-\mathrm{NMR}: \delta 56.7,77.0,77.5,112.3,123.4,123.9,126.6,127.6,129.2$, 131.9, 137.2, 141.9, 148.1, 152.8, 159.7. ESI-HRMS (m/z): calcd. for $\left(\mathrm{C}_{17} \mathrm{H}_{12} \mathrm{Cl}_{2} \mathrm{~N}_{3} \mathrm{O}_{3}\right)\left[\mathrm{M}+\mathrm{H}^{+}\right] 376.0251$, found 376.0260 .

(E)-1-(1-(5-Bromo-2-(prop-2-yn-1-yloxy)phenyl)-2,2-dichlorovinyl)-2-phenyldiazene (2e). Yield $271 \mathrm{mg}(66 \%)$, orange-red solid, m.p. $107^{\circ} \mathrm{C}$. IR $\left(v, \mathrm{~cm}^{-1}\right)$ : 1564, 1582, 1598, 3310. ${ }^{1} \mathrm{H}-\mathrm{NMR}: \delta 2.46(\mathrm{t}, 1 \mathrm{H}, J=2.4$ $\mathrm{Hz}), 4.60(\mathrm{~d}, 2 \mathrm{H}, J=2.4 \mathrm{~Hz}), 7.01(\mathrm{~d}, 1 \mathrm{H}, J=8.8 \mathrm{~Hz}), 7.26(\mathrm{~d}, 1 \mathrm{H}, J=2.5 \mathrm{~Hz}), 7.44-7.47(\mathrm{~m}, 3 \mathrm{H}), 7.52$ $(\mathrm{dd}, 1 \mathrm{H}, J=8.9,2.5 \mathrm{~Hz}), 7.76-7.80(\mathrm{~m}, 2 \mathrm{H}) .{ }^{13} \mathrm{C}-\mathrm{NMR}: 856.2,75.9,77.9,113.4,114.4,123.2,124.9,128.9$, 131.5, 133.0, 133.8, 136.5, 148.6, 152.8, 154.0. ESI-HRMS (m/z): calcd. for $\left(\mathrm{C}_{17} \mathrm{H}_{12} \mathrm{BrCl}_{2} \mathrm{~N}_{2} \mathrm{O}\right)\left[\mathrm{M}+\mathrm{H}^{+}\right]$ 408.9505, found 408.9520 .

(E)-1-(2,2-Dichloro-1-(3,5-di-tert-butyl-2-(prop-2-yn-1-yloxy)phenyl)vinyl)-2-phenyldiazene (2f). Yield 230 mg (52\%), orange-red oil. IR ( $\left.v, \mathrm{~cm}^{-1}\right): 1562,1599,3307 .{ }^{1} \mathrm{H}-\mathrm{NMR}: \delta 1.31(\mathrm{~s}, 9 \mathrm{H}), 1.45(\mathrm{~s}, 9 \mathrm{H}), 2.50(\mathrm{t}$, $1 \mathrm{H}, J=2.5 \mathrm{~Hz}), 4.19-4.38(\mathrm{~m}, 2 \mathrm{H}), 6.87(\mathrm{~d}, 1 \mathrm{H}, J=2.5 \mathrm{~Hz}), 7.41(\mathrm{~d}, 1 \mathrm{H}, J=2.5 \mathrm{~Hz}), 7.45-7.47(\mathrm{~m}, 3 \mathrm{H})$, 7.79-7.81 (m, 2H). ${ }^{13} \mathrm{C}-\mathrm{NMR}: \delta 30.3,31.0,34.2,34.8,60.6,74.6,78.7,122.9,124.5,125.0,126.3,128.6$, 131.1, 136.4, 141.4, 145.1, 150.7, 152.6, 154.0. ESI-HRMS (m/z): calcd. for $\left(\mathrm{C}_{25} \mathrm{H}_{29} \mathrm{Cl}_{2} \mathrm{~N}_{2} \mathrm{O}\right)\left[\mathrm{M}+\mathrm{H}^{+}\right]$ 443.1652, found 443.1649 .

(E)-1-(2,2-Dichloro-1-(2-(prop-2-yn-1-yloxy)naphthalen-1-yl)vinyl)-2-phenyldiazene (2g). Yield $175 \mathrm{mg}(46 \%)$, orange-red oil. IR $\left(v, \mathrm{~cm}^{-1}\right)$ : 1567, 1587, 1595, 3295. ${ }^{1} \mathrm{H}-\mathrm{NMR}: \delta 2.45(\mathrm{t}, 1 \mathrm{H}, J=2.4 \mathrm{~Hz}), 4.75(\mathrm{~d}, 2 \mathrm{H}, J=$ $2.4 \mathrm{~Hz}), 7.36-7.44(\mathrm{~m}, 6 \mathrm{H}), 7.47(\mathrm{~d}, 1 \mathrm{H}, J=9.1 \mathrm{~Hz}), 7.70-7.73(\mathrm{~m}, 2 \mathrm{H}), 7.85(\mathrm{~d}, 1 \mathrm{H}, J=7.7 \mathrm{~Hz}), 7.96(\mathrm{~d}$, $1 \mathrm{H}, J=9.1 \mathrm{~Hz}) .{ }^{13} \mathrm{C}-\mathrm{NMR}: \delta 57.0,75.6,78.8,114.6,117.9,123.2,124.1,124.2,127.2,128.2,128.8,129.2$, 131.0, 131.3, $132.1136 .9,148.2,152.7,153.0$. ESI-HRMS $(m / z)$ : calcd. for $\left(\mathrm{C}_{21} \mathrm{H}_{15} \mathrm{Cl}_{2} \mathrm{~N}_{2} \mathrm{O}\right)\left[\mathrm{M}+\mathrm{H}^{+}\right]$ 381.0556, found 381.0564 .

(E)-1-(2,2-Dichloro-1-(2-(prop-2-yn-1-yloxy)phenyl)vinyl)-2-(4-fluorophenyl)diazene (2h). Yield $175 \mathrm{mg}$ (50\%), orange-red solid, m.p. $60^{\circ} \mathrm{C}$. IR $\left(v, \mathrm{~cm}^{-1}\right)$ : 1574, 1592, 1603, 3301. ${ }^{1} \mathrm{H}-\mathrm{NMR}: \delta 2.44(\mathrm{t}, 1 \mathrm{H}, \mathrm{J}=$ $2.4 \mathrm{~Hz}), 4.63(\mathrm{~d}, 2 \mathrm{H}, J=2.4 \mathrm{~Hz}), 7.06-7.13(\mathrm{~m}, 5 \mathrm{H}), 7.41-7.45(\mathrm{~m}, 1 \mathrm{H}), 7.76-7.82(\mathrm{~m}, 2 \mathrm{H}) .{ }^{13} \mathrm{C}-\mathrm{NMR}: \delta$ 56.1, 75.5, 78.4, 112.7, 115.8, 116.0, 121.3, 122.8, 125.2, 125.3, 130.4, 131.3, 149.5, 149.7, 154.9, 163.3, 165.8 . ESI-HRMS $(\mathrm{m} / \mathrm{z})$ : calcd. for $\left(\mathrm{C}_{17} \mathrm{H}_{12} \mathrm{Cl}_{2} \mathrm{FN}_{2} \mathrm{O}\right)\left[\mathrm{M}+\mathrm{H}^{+}\right]$349.0305, found 349.0307. 
(E)-1-(2,2-Dichloro-1-(2-(prop-2-yn-1-yloxy)phenyl)vinyl)-2-(2,4-dichlorophenyl)diazene (2i). Yield $244 \mathrm{mg}$ (61\%), orange-red solid, m.p. $104{ }^{\circ} \mathrm{C}$. IR $\left(v, \mathrm{~cm}^{-1}\right)$ : 1575, 1599, 3300. ${ }^{1} \mathrm{H}-\mathrm{NMR}: \delta 2.44(\mathrm{t}, 1 \mathrm{H}, J=2.4 \mathrm{~Hz})$, $4.62(\mathrm{~d}, 2 \mathrm{H}, J=2.3 \mathrm{~Hz}), 7.05-7.15(\mathrm{~m}, 3 \mathrm{H}), 7.27-7.29(\mathrm{~m}, 1 \mathrm{H}), 7.40-7.44(\mathrm{~m}, 1 \mathrm{H}), 7.47(\mathrm{~d}, 1 \mathrm{H}, J=2.1 \mathrm{~Hz})$, $7.63(\mathrm{~d}, 1 \mathrm{H}, J=8.7 \mathrm{~Hz}) .{ }^{13} \mathrm{C}-\mathrm{NMR}: \delta 56.3,75.5,78.4,112.5,118.5,121.3,122.3,127.6,130.3,130.6,131.2$, 136.5, 137.4, 137.9, 147.6, 150.8, 155.1. ESI-HRMS $(\mathrm{m} / \mathrm{z})$ : calcd. for $\left(\mathrm{C}_{17} \mathrm{H}_{11} \mathrm{Cl}_{4} \mathrm{~N}_{2} \mathrm{O}\right)\left[\mathrm{M}+\mathrm{H}^{+}\right] 398.9620$, found 398.9622 .

(E)-1-(2-Chlorophenyl)-2-(2,2-dichloro-1-(2-(prop-2-yn-1-yloxy)phenyl)vinyl)diazene (2j). Yield $201 \mathrm{mg}$ (55\%), orange-red oil. IR $\left(v, \mathrm{~cm}^{-1}\right)$ : 1581, 1602, 3297. ${ }^{1} \mathrm{H}-\mathrm{NMR}: \delta 2.44(\mathrm{t}, 1 \mathrm{H}, J=2.4 \mathrm{~Hz}), 4.63(\mathrm{~d}, 2 \mathrm{H}, J=2.3$ $\mathrm{Hz}), 7.05-7.15(\mathrm{~m}, 3 \mathrm{H}), 7.30-7.38(\mathrm{~m}, 2 \mathrm{H}), 7.39-7.45(\mathrm{~m}, 2 \mathrm{H}), 7.65$ (dd, $1 \mathrm{H}, J=7.8,2.0 \mathrm{~Hz}) .{ }^{13} \mathrm{C}-\mathrm{NMR}: \delta$ 56.4, 75.5, 78.6, 112.6, 117.7, 121.3, 122.5, 127.1, 130.5, 130.6, 131.2, 131.9, 135.7, 137.3, 149.1, 150.6, 155.2. ESI-HRMS (m/z): calcd. for $\left(\mathrm{C}_{17} \mathrm{H}_{12} \mathrm{Cl}_{3} \mathrm{~N}_{2} \mathrm{O}\right)\left[\mathrm{M}+\mathrm{H}^{+}\right]$365.0010, found 365.0005.

(E)-1-(2,2-Dichloro-1-(2-(prop-2-yn-1-yloxy)phenyl)vinyl)-2-(2-methoxyphenyl)diazene (2k). Yield $235 \mathrm{mg}$ (65\%), orange-red solid, m.p. $102{ }^{\circ} \mathrm{C}$. IR $\left(v, \mathrm{~cm}^{-1}\right): 1570,1585,1594,1607,3235 .{ }^{1} \mathrm{H}-\mathrm{NMR}: \delta 2.44(\mathrm{t}$, $1 \mathrm{H}, J=2.4 \mathrm{~Hz}), 3.73(\mathrm{~s}, 3 \mathrm{H}), 4.61(\mathrm{~d}, 2 \mathrm{H}, J=2.5 \mathrm{~Hz}), 6.95-7.00(\mathrm{~m}, 2 \mathrm{H}), 7.07(\mathrm{td}, 1 \mathrm{H}, J=7.4,0.9 \mathrm{~Hz})$, 7.14-7.16 (m, 2H), 7.35-7.42 (m, 2H), $7.62(\mathrm{dd}, 1 \mathrm{H}, J=7.9,1.6 \mathrm{~Hz}) .{ }^{13} \mathrm{C}-\mathrm{NMR}: \delta 56.6,57.2,75.3,78.7$, 113.1, 114.3, 117.3, 120.9, 121.5, 123.1, 130.3, 131.3, 132.7, 135.2, 143.0, 150.3, 155.3, 157.3. ESI-HRMS (m/z): calcd. for $\left(\mathrm{C}_{18} \mathrm{H}_{15} \mathrm{Cl}_{2} \mathrm{~N}_{2} \mathrm{O}_{2}\right)\left[\mathrm{M}+\mathrm{H}^{+}\right] 361.0505$, found 361.0519.

(E)-1-(2,2-Dichloro-1-(2-(prop-2-yn-1-yloxy)phenyl)vinyl)-2-(4-methoxyphenyl)diazene (21). Yield $101 \mathrm{mg}$ (28\%), orange-red solid, m.p. $69^{\circ} \mathrm{C}$. IR $\left(v, \mathrm{~cm}^{-1}\right): 1569,1581,1601,3286 .{ }^{1} \mathrm{H}-\mathrm{NMR}: \delta 2.43(\mathrm{t}, 1 \mathrm{H}, J=2.4$ $\mathrm{Hz}), 3.86(\mathrm{~s}, 3 \mathrm{H}), 4.62(\mathrm{~d}, 2 \mathrm{H}, J=2.4 \mathrm{~Hz}), 6.92(\mathrm{dt}, 2 \mathrm{H}, J=9.8,2.6 \mathrm{~Hz}), 7.07(\mathrm{td}, 1 \mathrm{H}, J=7.3,0.9 \mathrm{~Hz})$, 7.10-7.14 (m, 2H), 7.39-7.44 (m, 1H), $7.77(\mathrm{dt}, 2 \mathrm{H}, J=9.7,2.6 \mathrm{~Hz}) .{ }^{13} \mathrm{C}-\mathrm{NMR}: \delta 55.5,56.2,75.4,78.6$, $112.8,114.0,121.3,123.3,125.2,130.2,131.4,133.9,147.4,149.7,155.0,162.3$. ESI-HRMS (m/z): calcd. for $\left(\mathrm{C}_{18} \mathrm{H}_{15} \mathrm{Cl}_{2} \mathrm{~N}_{2} \mathrm{O}_{2}\right)\left[\mathrm{M}+\mathrm{H}^{+}\right]$361.0505, found 361.0512.

(E)-1-(2,2-Dichloro-1-(2-(prop-2-yn-1-yloxy)phenyl)vinyl)-2-(2,4-dimethylphenyl)diazene (2m). Yield 320 mg $(89 \%)$, orange-red oil. IR $\left(v, \mathrm{~cm}^{-1}\right): 1575,1584,1606,3298 .{ }^{1} \mathrm{H}-\mathrm{NMR}: \delta 2.24(\mathrm{~s}, 3 \mathrm{H}), 2.35(\mathrm{~s}, 3 \mathrm{H})$, $2.44(\mathrm{t}, 1 \mathrm{H}, J=2.4 \mathrm{~Hz}), 4.62(\mathrm{~d}, 2 \mathrm{H}, J=2.4 \mathrm{~Hz}), 7.03-7.13(\mathrm{~m}, 5 \mathrm{H}), 7.39-7.43(\mathrm{~m}, 1 \mathrm{H}), 7.57-7.60(\mathrm{~m}$, 1H). ${ }^{13}$ C-NMR: $\delta$ 17.0, 21.4, 56.1, 75.5, 78.5, 112.4, 115.4, 121.1, 123.6, 127.1, 130.1, 131.2, 131.7, 134.2, 139.0, 141.9, 148.9, 150.4, 154.9. ESI-HRMS (m/z): calcd. for $\left(\mathrm{C}_{19} \mathrm{H}_{17} \mathrm{Cl}_{2} \mathrm{~N}_{2} \mathrm{O}\right)\left[\mathrm{M}+\mathrm{H}^{+}\right] 359.0712$, found 359.0715 .

(E)-1-(2,2-Dichloro-1-(2-(prop-2-yn-1-yloxy)phenyl)vinyl)-2-(2,6-dimethylphenyl)diazene (2n). Yield $320 \mathrm{mg}$ (89\%), orange-red oil. IR $\left(v, \mathrm{~cm}^{-1}\right): 1584,1605,3300 .{ }^{1} \mathrm{H}-\mathrm{NMR}: \delta 2.29(\mathrm{~s}, 6 \mathrm{H}), 2.47(\mathrm{t}, 1 \mathrm{H}, J=2.4 \mathrm{~Hz})$, $4.65(\mathrm{~s}, 2 \mathrm{H}), 7.09-7.18(\mathrm{~m}, 6 \mathrm{H}), 7.42-7.46(\mathrm{~m}, 1 \mathrm{H}) .{ }^{13} \mathrm{C}-\mathrm{NMR}: \delta 19.4,56.0,75.6,78.3,112.2,121.2,123.1$, 129.0, 129.1, 130.2, 131.1, 135.5, 150.5, 150.6, 154.7. ESI-HRMS $(\mathrm{m} / \mathrm{z})$ : calcd. for $\left(\mathrm{C}_{19} \mathrm{H}_{17} \mathrm{Cl}_{2} \mathrm{~N}_{2} \mathrm{O}\right)[\mathrm{M}+$ $\mathrm{H}^{+}$] 359.0712, found 359.0718 .

(E)-4-((2,2-Dichloro-1-(2-(prop-2-yn-1-yloxy)phenyl)vinyl)diazenyl)benzonitrile (2o). Yield $146 \mathrm{mg}(41 \%)$, orange-red solid, m.p. $154{ }^{\circ} \mathrm{C}$. IR $\left(v, \mathrm{~cm}^{-1}\right): 1578,1608,3303 .{ }^{1} \mathrm{H}-\mathrm{NMR}: \delta 2.44(\mathrm{t}, 1 \mathrm{H}, J=2.4 \mathrm{~Hz}), 4.63$ $(\mathrm{d}, 2 \mathrm{H}, J=2.3 \mathrm{~Hz}), 7.07-7.13(\mathrm{~m}, 3 \mathrm{H}), 7.42-7.47(\mathrm{~m}, 1 \mathrm{H}), 7.71-7.73(\mathrm{~m}, 2 \mathrm{H}), 7.82-7.84(\mathrm{~m}, 2 \mathrm{H}) .{ }^{13} \mathrm{C}-\mathrm{NMR}$ :

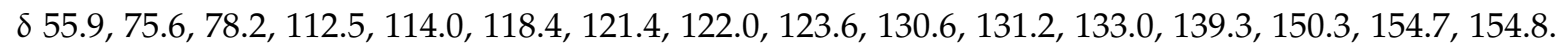
ESI-HRMS ( $m / z)$ : calcd. for $\left(\mathrm{C}_{18} \mathrm{H}_{12} \mathrm{Cl}_{2} \mathrm{~N}_{3} \mathrm{O}\right)\left[\mathrm{M}+\mathrm{H}^{+}\right]$356.0352, found 356.0347.

\subsection{General Procedure for Preparation of 4-Azido-2-phenyl-5-(2-(prop-2-yn-1-yloxy)phenyl)-2H-1,2,3-triazoles}

Attention! All manipulations with any azides demand significant care due to safety reasons! $o$-Propargyloxy-substituted 4-azido-2,5-diaryl-1,2,3-triazoles were obtained according to the procedure described in this paper [27]. A $20 \mathrm{~mL}$ screw neck vial was charged with DMSO (10 mL) and sodium azide ( 5 eq.), then corresponding dichlorodiazene ( $1 \mathrm{mmol}, 1$ eq.) was added dropwise over $15 \mathrm{~min}$. The resulting mixture was stirred at room temperature for $2 \mathrm{~h}$. Next, reaction mixture was poured into water $(100 \mathrm{~mL})$, extracted with DCM $(3 \times 20 \mathrm{~mL})$. Combined extract was washed 
with water $(3 \times 50 \mathrm{~mL})$, brine $(1 \times 30 \mathrm{~mL})$ and dried over sodium sulfate. Volatiles were removed in vacuum of the rotary evaporator, the residue was purified by column chromatography on silica gel using a mixture of hexane and DCM (3/1). Compounds $3 \mathbf{h}, 3 \mathbf{k}, 3 \mathbf{l}, 3 \mathrm{~m}$ and $\mathbf{3 n}$ were obtained as a mixture of two atropoisomers. Thus, the NMR spectra of these compounds have dual signals.

4-Azido-2-phenyl-5-(2-(prop-2-yn-1-yloxy)phenyl)-2H-1,2,3-triazole (3a). Yield $138 \mathrm{mg}$ (64\%), yellow oil. IR $\left(v, \mathrm{~cm}^{-1}\right): 3290,2131 .{ }^{1} \mathrm{H}-\mathrm{NMR}: \delta 2.55(\mathrm{t}, 1 \mathrm{H}, J=2.4 \mathrm{~Hz}), 4.80(\mathrm{~d}, 2 \mathrm{H}, J=2.4 \mathrm{~Hz}), 7.11-7.17(\mathrm{~m}, 2 \mathrm{H})$, 7.29-7.36 (m, 1H), 7.42-7.51 (m, 4H), 8.06-8.08 (m, 2H). ${ }^{13} \mathrm{C}-\mathrm{NMR}: \delta 56.1,75.5,78.5,112.7,121.3,122.9$, 123.2, 128.9, 130.3, 131.3, 131.3, 136.0, 149.9, 153.0, 154.9. ESI-HRMS $(\mathrm{m} / \mathrm{z})$ : calcd. for $\left(\mathrm{C}_{17} \mathrm{H}_{13} \mathrm{~N}_{6} \mathrm{O}\right)[\mathrm{M}+$ $\left.\mathrm{H}^{+}\right]$317.1146, found 317.1146.

4-Azido-5-(3-methoxy-2-(prop-2-yn-1-yloxy)phenyl)-2-phenyl-2H-1,2,3-triazole (3b). Yield $43 \mathrm{mg}(68 \%)$, white solid, m. p. $102{ }^{\circ} \mathrm{C}$. IR $\left(v, \mathrm{~cm}^{-1}\right)$ : 3296, 2125. ${ }^{1} \mathrm{H}-\mathrm{NMR}: \delta 2.42(\mathrm{t}, 1 \mathrm{H}, J=2.4 \mathrm{~Hz}), 3.92(\mathrm{~s}, 3 \mathrm{H}), 4.82$ $(\mathrm{d}, 2 \mathrm{H}, J=2.4 \mathrm{~Hz}), 7.01-7.03(\mathrm{~m}, 1 \mathrm{H}), 7.17-7.21(\mathrm{~m}, 2 \mathrm{H}), 7.35(\mathrm{t}, 1 \mathrm{H}, J=7.4 \mathrm{~Hz}), 7.49(\mathrm{t}, 2 \mathrm{H}, J=7.9 \mathrm{~Hz})$, $8.10(\mathrm{~d}, 2 \mathrm{H}, J=7.8 \mathrm{~Hz}) .{ }^{13} \mathrm{C}-\mathrm{NMR}: \delta 55.9,60.6,74.8,79.2,113.3,118.2,122.1,123.6,124.8,127.2,129.2$, 135.9, 139.5, 144.3, 144.8, 153.2. ESI-HRMS $(\mathrm{m} / \mathrm{z})$ : calcd. for $\left(\mathrm{C}_{18} \mathrm{H}_{15} \mathrm{~N}_{6} \mathrm{O}_{2}\right)\left[\mathrm{M}+\mathrm{H}^{+}\right]$347.1251, found 347.0787.

4-Azido-5-(3-nitro-2-(prop-2-yn-1-yloxy)phenyl)-2-phenyl-2H-1,2,3-triazole (3c). Yield $114 \mathrm{mg}$ (63\%), yellow solid, m. p. $120{ }^{\circ} \mathrm{C}$. IR $\left(v, \mathrm{~cm}^{-1}\right)$ : 3303, 2130. ${ }^{1} \mathrm{H}-\mathrm{NMR}: \delta 2.46(\mathrm{t}, 1 \mathrm{H}, J=2.4 \mathrm{~Hz}), 4.78(\mathrm{~d}, 2 \mathrm{H}, J=2.5 \mathrm{~Hz})$, 7.36-7.41 (m, 2H), $7.51(\mathrm{t}, 2 \mathrm{H}, J=7.9 \mathrm{~Hz}), 7.91(\mathrm{~d}, 2 \mathrm{H}, J=8.2 \mathrm{~Hz}), 8.10(\mathrm{~d}, 2 \mathrm{H}, J=8.2 \mathrm{~Hz}) .{ }^{13} \mathrm{C}-\mathrm{NMR}: \delta$ 63.1, 76.7, 77.5, 118.3, 125.0, 125.8, 126.1, 127.8, 129.4, 133.6, 135.2, 139.2, 144.7, 145.5, 148.8. ESI-HRMS $(\mathrm{m} / \mathrm{z})$ : calcd. for $\left(\mathrm{C}_{17} \mathrm{H}_{12} \mathrm{BrN}_{6} \mathrm{O}\right)\left[\mathrm{M}+\mathrm{H}^{+}\right]$395.0251, found 395.0250.

4-Azido-5-(5-nitro-2-(prop-2-yn-1-yloxy)phenyl)-2-phenyl-2H-1,2,3-triazole (3d). Yield $114 \mathrm{mg}$ (63\%), white-yellow solid, m. p. $160{ }^{\circ} \mathrm{C}$. IR $\left(v, \mathrm{~cm}^{-1}\right)$ : 3286, 2131. ${ }^{1} \mathrm{H}-\mathrm{NMR}: \delta 2.57(\mathrm{t}, 1 \mathrm{H}, J=2.4 \mathrm{~Hz}), 4.78(\mathrm{~d}$, $2 \mathrm{H}, J=2.4 \mathrm{~Hz}), 7.04(\mathrm{~d}, 1 \mathrm{H}, J=8.8 \mathrm{~Hz}), 7.34-7.37(\mathrm{~m}, 1 \mathrm{H}), 7.47-7.54(\mathrm{~m}, 3 \mathrm{H}), 7.72(\mathrm{~d}, 1 \mathrm{H}, J=2.5 \mathrm{~Hz})$, 8.05-8.07 (m, 2H). ${ }^{13} \mathrm{C}-\mathrm{NMR}: \delta 58.9,76.1,77.8,114.0,115.1,118.2,120.5,127.4,129.3,133.1,133.6,134.8$, 139.3, 144.4, 154.3. ESI-HRMS (m/z): calcd. for $\left(\mathrm{C}_{17} \mathrm{H}_{12} \mathrm{BrN}_{6} \mathrm{O}\right)\left[\mathrm{M}+\mathrm{H}^{+}\right]$395.0251, found 395.0250.

4-Azido-5-(5-bromo-2-(prop-2-yn-1-yloxy)phenyl)-2-phenyl-2H-1,2,3-triazole (3e). Yield $158 \mathrm{mg}(80 \%)$, yellow solid, m.p. $218^{\circ} \mathrm{C}$. IR $\left(v, \mathrm{~cm}^{-1}\right): 3292,2131 .{ }^{1} \mathrm{H}-\mathrm{NMR}: \delta 2.57(\mathrm{t}, 1 \mathrm{H}, J=2.4 \mathrm{~Hz}), 4.78(\mathrm{~d}, 2 \mathrm{H}, J=$ $2.4 \mathrm{~Hz}), 7.04(\mathrm{~d}, 1 \mathrm{H}, J=8.8 \mathrm{~Hz}), 7.34-7.37(\mathrm{~m}, 1 \mathrm{H}), 7.47-7.54(\mathrm{~m}, 3 \mathrm{H}), 7.72(\mathrm{~d}, 1 \mathrm{H}, J=2.5 \mathrm{~Hz}), 8.05-8.07$ (m, 2H). ${ }^{13}$ C-NMR: $\delta 58.9,76.1,77.8,114.0,115.1,118.2,120.5,127.4,129.3,133.1,133.6,134.8,139.3$, 144.4, 154.3. ESI-HRMS (m/z): calcd. for $\left(\mathrm{C}_{17} \mathrm{H}_{12} \mathrm{BrN}_{6} \mathrm{O}\right)\left[\mathrm{M}+\mathrm{H}^{+}\right]$395.0251, found 395.0250.

4-Azido-5-(3,5-di-tert-butyl-2-(prop-2-yn-1-yloxy)phenyl)-2-phenyl-2H-1,2,3-triazole (3f). Yield $231 \mathrm{mg}$ (59\%), white-yellow solid, m.p. $114{ }^{\circ} \mathrm{C} . \mathrm{IR}\left(\mathrm{v}, \mathrm{cm}^{-1}\right): 3310,2131 .{ }^{1} \mathrm{H}-\mathrm{NMR}: \delta 1.38(\mathrm{~s}, 9 \mathrm{H}), 1.51(\mathrm{~s}, 9 \mathrm{H})$, $2.42(\mathrm{t}, 1 \mathrm{H}, J=2.4 \mathrm{~Hz}), 4.28(\mathrm{dd}, 2 \mathrm{H}, J=14.9,2.4 \mathrm{~Hz}), 7.35-7.39(\mathrm{~m}, 2 \mathrm{H}), 7.49-7.53(\mathrm{~m}, 3 \mathrm{H}), 8.13(\mathrm{~d}, 2 \mathrm{H}, J$ $=7.7 \mathrm{~Hz}) .{ }^{13} \mathrm{C}-\mathrm{NMR}: \delta 31.0,31.4,34.6,35.3,61.2,74.9,78.7,118.2,118.5,125.9,126.6,127.3,127.8,129.2$, $137.5,139.4,142.7,144.2,146.4,153.8$. ESI-HRMS $(m / z)$ : calcd. for $\left(\mathrm{C}_{25} \mathrm{H}_{29} \mathrm{~N}_{6} \mathrm{O}\right)\left[\mathrm{M}+\mathrm{H}^{+}\right]$429.2397, found 429.2410 .

4-Azido-2-phenyl-5-(2-(prop-2-yn-1-yloxy)naphthalen-1-yl)-2H-1,2,3-triazole (3g). Yield $38 \mathrm{mg}$ (35\%), yellow oil. IR $\left(v, \mathrm{~cm}^{-1}\right): 2130 .{ }^{1} \mathrm{H}-\mathrm{NMR}: \delta 2.52(\mathrm{t}, 1 \mathrm{H}, J=2.5 \mathrm{~Hz}), 4.84(\mathrm{~d}, 2 \mathrm{H}, J=2.4 \mathrm{~Hz}), 7.37-7.55(\mathrm{~m}, 6 \mathrm{H})$, 7.66-7.68 (m, 1H), 7.88-7.90 (m, 1H), $8.03(\mathrm{~d}, 1 \mathrm{H}, J=9.1 \mathrm{~Hz}), 8.13-8.16(\mathrm{~m}, 2 \mathrm{H}) .{ }^{13} \mathrm{C}-\mathrm{NMR}: \delta 57.2,76.0$, 78.4, 111.8, 114.5, 118.4, 124.4, 127.4, 127.7, 128.2, 129.3, 131.7, 133.4, 139.4, 139.6, 154.1. ESI-HRMS (m/z): calcd. for $\left(\mathrm{C}_{21} \mathrm{H}_{15} \mathrm{~N}_{6} \mathrm{O}\right)\left[\mathrm{M}+\mathrm{H}^{+}\right]$367.1302, found 367.1309.

4-Azido-2-(4-fluorophenyl)-5-(2-(prop-2-yn-1-yloxy)phenyl)-2H-1,2,3-triazole (3h). Yield $155 \mathrm{mg}$ (93\%), yellow oil. IR $\left(v, \mathrm{~cm}^{-1}\right)$ : $2131 .{ }^{1} \mathrm{H}-\mathrm{NMR}: \delta 2.57(\mathrm{t}, 2 \mathrm{H}, J=2.3 \mathrm{~Hz}), 4.80(\mathrm{~d}, 4 \mathrm{H}, J=2.4 \mathrm{~Hz}), 7.11-7.21(\mathrm{~m}$, $8 \mathrm{H}), 7.42-7.51(\mathrm{~m}, 2 \mathrm{H}), 7.54-7.58(\mathrm{~m}, 2 \mathrm{H}), 8.01-8.07(\mathrm{~m}, 2 \mathrm{H}) .{ }^{13} \mathrm{C}-\mathrm{NMR}: \delta 56.1,56.5,75.7,75.8,78.2,78.2$, 112.9, 113.2, 115.9, 115.9, 116.1, 116.2, 117.9, 118.1, 119.7, 119.8, 120.0, 120.1, 121.5, 121.7, 130.7, 130.9, 131.1, 131.4, 135.7, 135.7, 136.3, 144.3, 155.2, 155.3, 160.3, 160.5, 162.7, 163.0. ESI-HRMS (m/z): calcd. for $\left(\mathrm{C}_{17} \mathrm{H}_{12} \mathrm{FN}_{6} \mathrm{O}\right)\left[\mathrm{M}+\mathrm{H}^{+}\right]$335.1052, found 335.1053. 
4-Azido-2-(2,4-dichlorophenyl)-5-(2-(prop-2-yn-1-yloxy)phenyl)-2H-1,2,3-triazole (3i). Yield $121 \mathrm{mg}(63 \%)$, yellow oil. IR $\left(v, \mathrm{~cm}^{-1}\right): 3298,2131 .{ }^{1} \mathrm{H}-\mathrm{NMR}: \delta 2.55(\mathrm{t}, 1 \mathrm{H}, J=2.4 \mathrm{~Hz}), 4.80(\mathrm{~d}, 2 \mathrm{H}, J=2.4 \mathrm{~Hz}), 7.10-7.17$ $(\mathrm{m}, 2 \mathrm{H}), 7.38-7.46(\mathrm{~m}, 2 \mathrm{H}), 7.56(\mathrm{dd}, 1 \mathrm{H}, J=7.6,1.7 \mathrm{~Hz}), 7.60(\mathrm{~d}, 1 \mathrm{H}, J=2.3 \mathrm{~Hz}), 7.66(\mathrm{~d}, 1 \mathrm{H}, J=8.6 \mathrm{~Hz})$. ${ }^{13} \mathrm{C}-\mathrm{NMR}\left(100 \mathrm{MHz}, \mathrm{CDCl}_{3}\right): \delta 56.7,75.8,78.3,113.3,118.0,121.8,127.7,127.8,129.6,130.8,131.0,131.2$, 135.1, 136.3, 136.9, 144.8, 155.3. ESI-HRMS $(\mathrm{m} / \mathrm{z})$ : calcd. for $\left(\mathrm{C}_{17} \mathrm{H}_{11} \mathrm{Cl}_{2} \mathrm{~N}_{6} \mathrm{O}\right)\left[\mathrm{M}+\mathrm{H}^{+}\right] 385.0366$, found 385.0363.

4-Azido-2-(2-chlorophenyl)-5-(2-(prop-2-yn-1-yloxy)phenyl)-2H-1,2,3-triazole (3j). Yield $109 \mathrm{mg}$ (62\%), yellow solid, m.p. $162{ }^{\circ} \mathrm{C}$. IR $\left(v, \mathrm{~cm}^{-1}\right)$ : 3296, 2128. ${ }^{1} \mathrm{H}-\mathrm{NMR}: \delta 2.55(\mathrm{t}, 1 \mathrm{H}, J=2.4 \mathrm{~Hz}), 4.81(\mathrm{~d}, 2 \mathrm{H}$, $J=2.4 \mathrm{~Hz}), 7.10-7.18(\mathrm{~m}, 2 \mathrm{H}), 7.38-7.46(\mathrm{~m}, 3 \mathrm{H}), 7.56-7.59(\mathrm{~m}, 2 \mathrm{H}), 7.69-7.71(\mathrm{~m}, 1 \mathrm{H}) .{ }^{13} \mathrm{C}-\mathrm{NMR}: \delta$ 56.7, 75.7, 78.4, 113.4, 118.2, 121.8, 127.3, 127.4, 129.1, 130.0, 130.7, 131.1, 131.3, 136.5, 137.7, 144.5, 155.3 . ESI-HRMS $(m / z)$ : calcd. for $\left(\mathrm{C}_{17} \mathrm{H}_{12} \mathrm{ClN}_{6} \mathrm{O}\right)\left[\mathrm{M}+\mathrm{H}^{+}\right]$351.0756, found 351.0754.

4-Azido-2-(2-methoxyphenyl)-5-(2-(prop-2-yn-1-yloxy)phenyl)-2H-1,2,3-triazole (3k). Yield $149 \mathrm{mg}(86 \%)$, yellow oil. IR $\left(v, \mathrm{~cm}^{-1}\right)$ : 3292, 2132. ${ }^{1} \mathrm{H}-\mathrm{NMR}: \delta 2.55(\mathrm{t}, 2 \mathrm{H}, J=2.4 \mathrm{~Hz}), 3.90(\mathrm{~s}, 6 \mathrm{H}), 4.79(\mathrm{~d}, 4 \mathrm{H}, J=2.5$ HZ), 7.05-7.20 (m, 8H), 7.39-7.48 (m, 4H), 7.55-7.60 (m, 4H). ${ }^{13} \mathrm{C}-\mathrm{NMR}: \delta 56.2,56.3,75.7,75.7,78.4$, 78.4, 112.7, 112.8, 113.0, 113.3, 118.2, 118.5, 120.5, 120.6, 121.5, 121.7, 126.9, 129.4, 129.5, 130.4, 130.4, 130.6, 130.7, 131.3, 131.6, 135.9, 137.1, 142.7, 143.8, 153.3, 153.4, 155.3, 155.4. ESI-HRMS (m/z): calcd. for $\left(\mathrm{C}_{18} \mathrm{H}_{15} \mathrm{~N}_{6} \mathrm{O}_{2}\right)\left[\mathrm{M}+\mathrm{H}^{+}\right]$347.1251, found 347.1257.

4-Azido-2-(4-methoxyphenyl)-5-(2-(prop-2-yn-1-yloxy)phenyl)-2H-1,2,3-triazole (31). Yield $102 \mathrm{mg}$ (59\%), yellow oil. IR $\left(v, \mathrm{~cm}^{-1}\right): 3292,2131 .{ }^{1} \mathrm{H}-\mathrm{NMR}: \delta 2.55(\mathrm{t}, 1 \mathrm{H}, J=2.3 \mathrm{~Hz}), 2.57(\mathrm{t}, 1 \mathrm{H}, J=2.3 \mathrm{~Hz}), 3.83(\mathrm{~s}$, $6 \mathrm{H}), 4.78(\mathrm{~d}, 2 \mathrm{H}, J=2.4 \mathrm{~Hz}), 4.79(\mathrm{~d}, 2 \mathrm{H}, J=2.4 \mathrm{~Hz}), 6.97-7.01(\mathrm{~m}, 4 \mathrm{H}), 7.10-7.20(\mathrm{~m}, 4 \mathrm{H}), 7.40-7.49(\mathrm{~m}$, 2H), 7.55-7.60 (m, 2H), 7.96-8.01 (m, 4H). ${ }^{13} \mathrm{C}-\mathrm{NMR}: \delta 55.4,56.1,56.5,75.6,75.7,78.2,78.3,112.9,113.2$, $114.2,114.2,118.1,118.4,119.5,119.7,121.4,121.6,130.4,130.7,131.0,131.3,133.1,133.2,133.7,134.0$, $135.5,137.0,142.5,143.6,155.2,155.3,158.7,158.9$. ESI-HRMS $(\mathrm{m} / \mathrm{z})$ : calcd. for $\left(\mathrm{C}_{18} \mathrm{H}_{15} \mathrm{~N}_{6} \mathrm{O}_{2}\right)\left[\mathrm{M}+\mathrm{H}^{+}\right]$ 347.1251, found 347.1251.

4-Azido-2-(2,4-dimethylphenyl)-5-(2-(prop-2-yn-1-yloxy)phenyl)-2H-1,2,3-triazole (3m). Yield $162 \mathrm{mg}(56 \%)$, yellow oil. IR $\left(v, \mathrm{~cm}^{-1}\right)$ : 3292, 2119. ${ }^{1} \mathrm{H}-\mathrm{NMR}: \delta 2.39(\mathrm{~s}, \mathrm{H}), 2.45-2.47(\mathrm{~m}, 6 \mathrm{H}), 2.53(\mathrm{t}, 1 \mathrm{H}, J=2.4 \mathrm{~Hz})$, $2.55(\mathrm{t}, 1 \mathrm{H}, J=2.4 \mathrm{~Hz}), 4.79(\mathrm{~d}, 2 \mathrm{H}, J=2.4 \mathrm{~Hz}), 4.80(\mathrm{~d}, 2 \mathrm{H}, J=2.5 \mathrm{~Hz}), 7.09-7.21(\mathrm{~m}, 8 \mathrm{H}), 7.40-7.49$ (m, 2H), 7.51-7.58 (m, 4H). ${ }^{13} \mathrm{C}-\mathrm{NMR}: \delta 18.9,19.1,21.0,21.0,56.3,56.7,75.6,75.7,78.3,78.4,113.1$, 113.3, 118.3, 118.6, 121.5, 121.7, 124.6, 124.8, 127.2, 130.4, 130.7, 131.2, 131.5, 132.0, 132.2, 132.3, 132.3, 136.7, 137.0, 138.5, 138.9, 142.3, 143.5, 155.3, 155.4. ESI-HRMS $(\mathrm{m} / \mathrm{z})$ : calcd. for $\left(\mathrm{C}_{19} \mathrm{H}_{17} \mathrm{~N}_{6} \mathrm{O}\right)\left[\mathrm{M}+\mathrm{H}^{+}\right]$ 345.1459 , found 345.1458 .

4-Azido-2-(2,6-dimethylphenyl)-5-(2-(prop-2-yn-1-yloxy)phenyl)-2H-1,2,3-triazole (3n). Yield $168 \mathrm{mg}$ (58\%), yellow oil. IR $\left(v, \mathrm{~cm}^{-1}\right)$ : 3291, 2126. ${ }^{1} \mathrm{H}-\mathrm{NMR}$ : $\delta 2.14(\mathrm{~s}, 6 \mathrm{H}), 2.16(\mathrm{~s}, 6 \mathrm{H}), 2.52(\mathrm{t}, 1 \mathrm{H}, J=2.4 \mathrm{~Hz}), 2.53(\mathrm{t}$, $1 \mathrm{H}, J=2.4 \mathrm{~Hz}), 4.78(\mathrm{dd}, 2 \mathrm{H}, J=2.5 \mathrm{~Hz}), 4.79(\mathrm{dd}, 2 \mathrm{H}, J=2.5 \mathrm{~Hz}), 7.09-7.20(\mathrm{~m}, 8 \mathrm{H}), 7.28-7.33(\mathrm{~m}$, 2H), 7.40-7.49 (m, 2H), $7.56(\mathrm{t}, 1 \mathrm{H}, J=1.7 \mathrm{~Hz}), 7.58(\mathrm{t}, 1 \mathrm{H}, J=1.7 \mathrm{~Hz}) .{ }^{13} \mathrm{C}-\mathrm{NMR}: \delta 17.4,17.5,56.4$, 56.7, 75.6, 75.7, 78.3, 78.4, 113.2, 113.4, 118.3, 118.5, 121.5, 121.7, 128.2, 128.3, 129.8, 129.9, 130.4, 130.7, 131.1, 131.4, 135.1, 135.9, 136.0, 136.4, 139.1, 139.2, 142.1, 143.4, 155.3, 155.4. ESI-HRMS (m/z): calcd. for $\left(\mathrm{C}_{19} \mathrm{H}_{17} \mathrm{~N}_{6} \mathrm{O}\right)\left[\mathrm{M}+\mathrm{H}^{+}\right] 345.1459$, found 345.1459.

4-(4-Azido-5-(2-(prop-2-yn-1-yloxy)phenyl)-2H-1,2,3-triazol-2-yl)benzonitrile (3o). Yield $102 \mathrm{mg}$ (60\%), grey solid, m. p. $124{ }^{\circ} \mathrm{C}$. IR $\left(v, \mathrm{~cm}^{-1}\right)$ : 3256, 2133. ${ }^{1} \mathrm{H}-\mathrm{NMR}: \delta 2.56(\mathrm{t}, 1 \mathrm{H}, J=2.4 \mathrm{~Hz}), 4.80(\mathrm{~d}, 2 \mathrm{H}, J=2.4 \mathrm{~Hz})$, 7.11-7.18 (m, 2H), 7.45-7.49 (m, 1H), $7.56(\mathrm{dd}, 1 \mathrm{H}, J=7.6,1.7 \mathrm{~Hz}), 7.77(\mathrm{dt}, 2 \mathrm{H}, J=9.0,2.0 \mathrm{~Hz}), 8.17(\mathrm{dt}$, $2 \mathrm{H}, J=9.0,2.0 \mathrm{~Hz}) .{ }^{13} \mathrm{C}-\mathrm{NMR}: \delta 56.6,75.8,78.1,110.3,113.2,117.6,118.3,118.3,121.8,131.1,131.2,133.5$, 138.0, 141.9, 145.9, 155.4. ESI-HRMS $(\mathrm{m} / \mathrm{z})$ : calcd. for $\left(\mathrm{C}_{18} \mathrm{H}_{12} \mathrm{~N}_{7} \mathrm{O}\right)\left[\mathrm{M}+\mathrm{H}^{+}\right] 342.1098$, found 342.1099.

3.4. General Procedure for Preparation of

2-Aryl-2H,8H-benzo[g]bis([1,2,3]triazolo)[5,1-c:4',5'-e][1,4]-oxazocines 4

To $3 \mathrm{~mL}$ of argon-sparged $o$-xylene the previously synthesized 4-azido-2-phenyl-5-(2-(prop-2-yn-1-yloxy)phenyl)-2H-1,2,3-triazole was added. The reaction 
mixture was refluxed under Ar for $12 \mathrm{~h}$ or more until TLC analysis showed complete consumption of source reagent. The final reaction mixture was evaporate and the residue was purified by column chromatography on silica gel using a mixture of hexane and EtOAc (6/1) as eluent.

2-Phenyl-2H,8H-benzo[g]bis([1,2,3]triazolo)[5,1-c:4',5'-e][1,4]oxazocine (4a). Yield $222 \mathrm{mg}$ (70\%), white solid, m. p. $157^{\circ} \mathrm{C}$. IR $\left(v, \mathrm{~cm}^{-1}\right)$ : no signals of azido group and triple bond. ${ }^{1} \mathrm{H}-\mathrm{NMR}: \delta 5.58(\mathrm{~s}, 2 \mathrm{H})$, 7.26-7.30 (m, 2H), $7.40(\mathrm{t}, 1 \mathrm{H}, J=7.4 \mathrm{~Hz}), 7.45-7.54(\mathrm{~m}, 3 \mathrm{H}), 7.63(\mathrm{~s}, 1 \mathrm{H}), 7.71(\mathrm{dd}, 1 \mathrm{H}, J=8.1,1.6$ $\mathrm{Hz}), 8.19-8.21(\mathrm{~m}, 2 \mathrm{H}) .{ }^{13} \mathrm{C}$ NMR $\left(100 \mathrm{MHz}, \mathrm{CDCl}_{3}\right): \delta 67.0,118.8,121.4,121.6,125.4,128.3,129.3$, $129.9,131.6,131.8,134.7,138.3,139.1,141.6,155.6$. ESI-HRMS $(m / z)$ : calcd. for $\left(\mathrm{C}_{17} \mathrm{H}_{13} \mathrm{~N}_{6} \mathrm{O}\right)\left[\mathrm{M}+\mathrm{H}^{+}\right]$ 317.1145, found 317.1144.

10-Methoxy-2-phenyl-2H,8H-benzo[g]bis([1,2,3]triazolo)[5,1-c:4',5'-e][1,4]oxazocine (4b). Yield $291 \mathrm{mg}$ $(84 \%)$, white-yellow solid, m. p. $170{ }^{\circ} \mathrm{C}$. IR $\left(v, \mathrm{~cm}^{-1}\right)$ : no signals of azido group and triple bond were observed. ${ }^{1} \mathrm{H}-\mathrm{NMR}: \delta 3.95(\mathrm{~s}, 3 \mathrm{H}), 5.65(\mathrm{~s}, 2 \mathrm{H}), 7.02-7.06(\mathrm{~m}, 1 \mathrm{H}), 7.22-7.25(\mathrm{~m}, 2 \mathrm{H}), 7.42(\mathrm{tt}, 1 \mathrm{H}, J$ $=7.4,1.3 \mathrm{~Hz}), 7.51-7.55(\mathrm{~m}, 2 \mathrm{H}), 7.62(\mathrm{~s}, 1 \mathrm{H}), 8.19-8.23(\mathrm{~m}, 2 \mathrm{H}) .{ }^{13} \mathrm{C}-\mathrm{NMR}: \delta 55.9,65.4,113.5,118.9$, $120.8,123.6,126.2,128.2,129.3,131.5,135.5,138.4,139.3,142.2,143.7,152.6$. ESI-HRMS $(\mathrm{m} / \mathrm{z})$ : calcd. for $\left(\mathrm{C}_{18} \mathrm{H}_{15} \mathrm{~N}_{6} \mathrm{O}\right)\left[\mathrm{M}+\mathrm{H}^{+}\right]$347.1251, found 347.1247.

10-Nitro-2-phenyl-2H,8H-benzo[g]bis([1,2,3]triazolo)[5,1-c:4',5'-e][1,4]oxazocine (4c). Yield $264 \mathrm{mg}(73 \%)$, white solid, m. p. $250^{\circ} \mathrm{C}$. IR $\left(v, \mathrm{~cm}^{-1}\right)$ : no signals of azido group and triple bond were observed. ${ }^{1} \mathrm{H}-\mathrm{NMR}$ (DMSO-d $\left.\mathrm{D}_{6}\right): \delta 5.83(\mathrm{~s}, 2 \mathrm{H}), 7.55-7.59(\mathrm{~m}, 2 \mathrm{H}), 7.68(\mathrm{t}, 2 \mathrm{H}, J=7.9 \mathrm{~Hz}), 8.03(\mathrm{~s}, 1 \mathrm{H}), 8.17(\mathrm{~d}, 2 \mathrm{H}, J$ $=7.9 \mathrm{~Hz}), 8.23(\mathrm{~d}, 2 \mathrm{H}, J=8.0 \mathrm{~Hz}) .{ }^{13} \mathrm{C}-\mathrm{NMR}\left(\mathrm{DMSO}_{\mathrm{d}}\right): \delta 66.2,118.9,123.8,125.9,127.6,129.2,130.1$, $133.3,134.7,135.7,136.6,138.5,140.9,143.5,148.3$. ESI-HRMS $(\mathrm{m} / \mathrm{z})$ : calcd. for $\left(\mathrm{C}_{17} \mathrm{H}_{12} \mathrm{~N}_{7} \mathrm{O}_{3}\right)\left[\mathrm{M}+\mathrm{H}^{+}\right]$ 362.0996, found 362.0997 .

12-Nitro-2-phenyl-2H,8H-benzo[g]bis([1,2,3]triazolo)[5,1-c:4',5'-e][1,4]oxazocine (4d). Yield $246 \mathrm{mg}(68 \%)$, white solid, m. p. $237^{\circ} \mathrm{C}$. IR $\left(v, \mathrm{~cm}^{-1}\right)$ : no signals of azido group and triple bond were observed. ${ }^{1} \mathrm{H}-\mathrm{NMR}: \delta 5.58(\mathrm{~s}, 2 \mathrm{H}), 7.34(\mathrm{~d}, 1 \mathrm{H}, J=9.1 \mathrm{~Hz}), 7.47-7.51(\mathrm{~m}, 1 \mathrm{H}), 7.58(\mathrm{t}, 2 \mathrm{H}, J=7.8 \mathrm{~Hz}), 7.86(\mathrm{~s}, 1 \mathrm{H})$, 8.23-8.25 (m, 2H), $8.29(\mathrm{dd}, 1 \mathrm{H}, J=9.1,2.8 \mathrm{~Hz}), 8.78(\mathrm{~d}, 1 \mathrm{H}, J=2.8 \mathrm{~Hz}) .{ }^{13} \mathrm{C}-\mathrm{NMR}: \delta 63.5,110.6,119.1$, 122.9, 126.4, 127.6, 129.1, 129.6, 133.0, 133.3, 136.7, 138.9, 144.0, 159.8, 160.6, 169.7, 178.8. ESI-HRMS (m/z): calcd. for $\left(\mathrm{C}_{17} \mathrm{H}_{12} \mathrm{~N}_{7} \mathrm{O}_{3}\right)\left[\mathrm{M}+\mathrm{H}^{+}\right]$362.0996, found 362.1008.

12-Bromo-2-phenyl-2H,8H-benzo[g]bis([1,2,3]triazolo)[5,1-c:4',5'-e][1,4]oxazocine (4e). Yield $261 \mathrm{mg}(66 \%)$, white solid, m. p. $224^{\circ} \mathrm{C}$. IR $\left(v, \mathrm{~cm}^{-1}\right)$ : no signals of azido group and triple bond were observed. ${ }^{1} \mathrm{H}-\mathrm{NMR}: \delta 5.56(\mathrm{~s}, 2 \mathrm{H}), 7.18(\mathrm{~d}, 1 \mathrm{H}, J=8.7 \mathrm{~Hz}), 7.45(\mathrm{tt}, 1 \mathrm{H}, J=7.4,1.3 \mathrm{~Hz}), 7.53-7.60(\mathrm{~m}, 3 \mathrm{H}), 7.68(\mathrm{~s}$, $1 \mathrm{H}), 7.89(\mathrm{~d}, 1 \mathrm{H}, \mathrm{J}=2.5 \mathrm{~Hz}), 8.19-8.22(\mathrm{~m}, 2 \mathrm{H}) .{ }^{13} \mathrm{C}-\mathrm{NMR}: \delta 66.6,118.2,118.9,123.3,123.4,128.6,129.5$, $131.9,132.8,134.2,134.6,137.0,139.1,141.5,154.7$. ESI-HRMS $(\mathrm{m} / \mathrm{z})$ : calcd. for $\left(\mathrm{C}_{17} \mathrm{H}_{12} \mathrm{BrN}_{6} \mathrm{O}\right)[\mathrm{M}+$ $\left.\mathrm{H}^{+}\right]$395.0250, found 395.0252.

10,12-Di-tert-butyl-2-phenyl-2H,8H-benzo[g]bis([1,2,3]triazolo)[5,1-c:4',5'-e][1,4]oxazocine (4f). Yield 335 $\mathrm{mg}(78 \%)$, white-yellow solid, m. p. $217^{\circ} \mathrm{C}$. IR $\left(v, \mathrm{~cm}^{-1}\right)$ : no signals of azido group and triple bond were observed. ${ }^{1} \mathrm{H}-\mathrm{NMR}: \delta 1.37(\mathrm{~s}, 9 \mathrm{H}), 1.47(\mathrm{~s}, 9 \mathrm{H}), 5.39(\mathrm{~s}, 2 \mathrm{H}), 7.44(\mathrm{t}, 1 \mathrm{H}, J=7.4 \mathrm{~Hz}), 7.51-7.57(\mathrm{~m}$, $3 \mathrm{H}), 7.77(\mathrm{~s}, 1 \mathrm{H}), 7.86(\mathrm{~d}, 1 \mathrm{H}, J=2.5 \mathrm{~Hz}), 8.23-8.25(\mathrm{~m}, 2 \mathrm{H}) .{ }^{13} \mathrm{C}-\mathrm{NMR}: \delta 31.3,34.7,35.5,65.2,119.0$, 121.2, 126.4, 126.9, 128.4, 129.4, 132.6, 134.2, 138.4, 139.1, 140.0, 142.1, 146.8, 153.8. ESI-HRMS (m/z): calcd. for $\left(\mathrm{C}_{25} \mathrm{H}_{29} \mathrm{~N}_{6} \mathrm{O}\right)\left[\mathrm{M}+\mathrm{H}^{+}\right]$429.2397, found 429.2402 .

14-Phenyl-8H,14H-naphtho[1-g]bis([1,2,3]triazolo)[5,1-c:4',5'-e][1,4]oxazocine (4g). Yield $184 \mathrm{mg}(50 \%)$, white-yellow solid, m. p. $143{ }^{\circ} \mathrm{C}$. IR $\left(v, \mathrm{~cm}^{-1}\right)$ : no signals of azido group and triple bond were observed. ${ }^{1} \mathrm{H}-\mathrm{NMR}: \delta 5.69(\mathrm{~s}, 2 \mathrm{H}), 7.43-7.47(\mathrm{~m}, 2 \mathrm{H}), 7.51-7.61(\mathrm{~m}, 5 \mathrm{H}), 7.88(\mathrm{~d}, 1 \mathrm{H}, J=7.8 \mathrm{~Hz}), 8.01(\mathrm{~d}, 1 \mathrm{H}, J=$ $8.7 \mathrm{~Hz}), 8.28-8.31(\mathrm{~m}, 2 \mathrm{H}), 8.33(\mathrm{~d}, 1 \mathrm{H}, J=8.5 \mathrm{~Hz}) .{ }^{13} \mathrm{C}-\mathrm{NMR}: \delta 67.4,111.3,117.0,118.9,119.5,125.2$, 126.1, 128.0, 128.3, 129.4, 131.3, 131.3, 132.7, 134.9, 137.2, 139.4, 143.2, 153.6. ESI-HRMS (m/z): calcd. for $\left(\mathrm{C}_{21} \mathrm{H}_{15} \mathrm{~N}_{6} \mathrm{O}\right)\left[\mathrm{M}+\mathrm{H}^{+}\right]$367.1302, found 367.1309.

2-(4-Fluorophenyl)-2H,8H-benzo[g]bis([1,2,3]triazolo)[5,1-c:4',5'-e][1,4]oxazocine (4h). Yield $285 \mathrm{mg}(85 \%)$, white solid, m. p. $168^{\circ} \mathrm{C}$. IR $\left(v, \mathrm{~cm}^{-1}\right)$ : no signals of azido group and triple bond were observed. 
${ }^{1} \mathrm{H}-\mathrm{NMR}: \delta 5.59(\mathrm{~s}, 2 \mathrm{H}), 7.18-7.24(\mathrm{~m}, 2 \mathrm{H}), 7.27-7.31(\mathrm{~m}, 2 \mathrm{H}), 7.46-7.51(\mathrm{~m}, 1 \mathrm{H}), 7.64(\mathrm{~s}, 1 \mathrm{H}), 7.68-7.70$ (m, 1H), 8.15-8.20 (m, 2H). ${ }^{13} \mathrm{C}-\mathrm{NMR}: \delta 67.1,116.1,116.4,120.6,120.7,121.5,125.5,129.9,131.6,131.9$, 134.7, 135.5, 138.4, 141.7, 155.7, 161.0, 163.4. ESI-HRMS $(\mathrm{m} / \mathrm{z})$ : calcd. for $\left(\mathrm{C}_{17} \mathrm{H}_{12} \mathrm{FN}_{6} \mathrm{O}\right)\left[\mathrm{M}+\mathrm{H}^{+}\right]$ 335.1051 , found 335.1050 .

2-(2,4-Dichlorophenyl)-2H,8H-benzo[g]bis([1,2,3]triazolo)[5,1-c:4',5'-e][1,4]oxazocine (4i). Yield $331 \mathrm{mg}$ $(86 \%)$, white-yellow solid, m. p. $110^{\circ} \mathrm{C}$. IR $\left(v, \mathrm{~cm}^{-1}\right)$ : no signals of azido group and triple bond were observed. ${ }^{1} \mathrm{H}-\mathrm{NMR}: \delta 5.61(\mathrm{~s}, 2 \mathrm{H}), 7.27-7.32(\mathrm{~m}, 2 \mathrm{H}), 7.43-7.52(\mathrm{~m}, 2 \mathrm{H}), 7.63-7.64(\mathrm{~m}, 2 \mathrm{H}), 7.69(\mathrm{dd}, 1 \mathrm{H}$, $J=7.6,1.4 \mathrm{~Hz}), 7.77(\mathrm{~d}, 1 \mathrm{H}, J=8.6 \mathrm{~Hz}) .{ }^{13} \mathrm{C}-\mathrm{NMR}: \delta 67.3,121.5,121.5,125.6,127.8,128.2,130.0,130.0$, 131.0, 131.6, 132.0, 134.7, 136.0, 136.0, 138.9, 142.2, 155.7. ESI-HRMS $(\mathrm{m} / \mathrm{z})$ : calcd. for $\left(\mathrm{C}_{17} \mathrm{H}_{11} \mathrm{Cl}_{2} \mathrm{~N}_{6} \mathrm{O}\right)$ $\left[\mathrm{M}+\mathrm{H}^{+}\right]$385.0366, found 385.0373.

2-(2-Chlorophenyl)-2H,8H-benzo[g]bis([1,2,3]triazolo)[5,1-c:4',5'-e][1,4]oxazocine (4j). Yield $242 \mathrm{mg}(69 \%)$, white solid, m. p. $80{ }^{\circ} \mathrm{C}$. IR $\left(v, \mathrm{~cm}^{-1}\right)$ : no signals of azido group and triple bond were observed. ${ }^{1} \mathrm{H}-\mathrm{NMR}: \delta 5.63(\mathrm{~s}, 2 \mathrm{H}), 7.28-7.33(\mathrm{~m}, 2 \mathrm{H}), 7.45-7.53(\mathrm{~m}, 3 \mathrm{H}), 7.61-7.65(\mathrm{~m}, 2 \mathrm{H}), 7.73(\mathrm{dd}, 1 \mathrm{H}, J=7.6,1.6$ $\mathrm{Hz}), 7.80-7.84(\mathrm{~m}, 1 \mathrm{H}) .{ }^{13} \mathrm{C}-\mathrm{NMR}: \delta 67.2,121.5,121.6,125.6,127.5,127.7,129.5,130.1,130.8,131.2,131.6$, 131.9, 134.7, 137.5, 138.6, 142.0, 155.7. ESI-HRMS $(\mathrm{m} / \mathrm{z})$ : calcd. for $\left(\mathrm{C}_{17} \mathrm{H}_{12} \mathrm{ClN} \mathrm{N}_{6} \mathrm{O}\right)\left[\mathrm{M}+\mathrm{H}^{+}\right] 351.0756$, found 351.0758 .

2-(2-Methoxyphenyl)-2H,8H-benzo[g]bis([1,2,3]triazolo)[5,1-c:4',5'-e][1,4]oxazocine (4k). Yield $232 \mathrm{mg}$ $(67 \%)$, white solid, m. p. $65^{\circ} \mathrm{C}$. IR $\left(v, \mathrm{~cm}^{-1}\right)$ : no signals of azido group and triple bond were observed. ${ }^{1} \mathrm{H}-\mathrm{NMR}: \delta 3.89(\mathrm{~s}, 3 \mathrm{H}), 5.58(\mathrm{~s}, 2 \mathrm{H}), 7.08-7.12(\mathrm{~m}, 2 \mathrm{H}), 7.23-7.29(\mathrm{~m}, 2 \mathrm{H}), 7.43-7.50(\mathrm{~m}, 2 \mathrm{H}), 7.62(\mathrm{~s}, 1 \mathrm{H})$, 7.68-7.72 (m, 2H). ${ }^{13} \mathrm{C}-\mathrm{NMR}: \delta 56.2,67.0,112.6,120.5,121.4,121.8,125.4,127.1,129.0,130.0,131.1,131.5$, 131.6, 134.5, 137.9, 141.4, 153.5, 155.6. ESI-HRMS (m/z): calcd. for $\left(\mathrm{C}_{18} \mathrm{H}_{15} \mathrm{~N}_{6} \mathrm{O}_{2}\right)\left[\mathrm{M}+\mathrm{H}^{+}\right]$347.1251, found 347.1244 .

2-(4-Methoxyphenyl)-2H,8H-benzo[g]bis([1,2,3]triazolo)[5,1-c:4',5'-e][1,4]oxazocine (41). Yield $253 \mathrm{mg}(73 \%)$, white solid, m. p. $65{ }^{\circ} \mathrm{C}$. IR $\left(v, \mathrm{~cm}^{-1}\right)$ : no signals of azido group and triple bond were observed. ${ }^{1} \mathrm{H}-\mathrm{NMR}: \delta 3.89(\mathrm{~s}, 3 \mathrm{H}), 5.59(\mathrm{~s}, 2 \mathrm{H}), 7.02-7.06(\mathrm{~m}, 2 \mathrm{H}), 7.28-7.31(\mathrm{~m}, 2 \mathrm{H}), 7.46-7.51(\mathrm{~m}, 1 \mathrm{H}), 7.65(\mathrm{~s}, 1 \mathrm{H})$, $7.72(\mathrm{dd}, 1 \mathrm{H}, J=8.0,1.8 \mathrm{~Hz}), 8.11-8.15(\mathrm{~m}, 2 \mathrm{H}) .{ }^{13} \mathrm{C}-\mathrm{NMR}: \delta 55.6,67.0,114.4,120.4,121.5,121.8,125.5$, $130.1,131.7,131.7,133.0,134.6,137.8,141.2,155.6,159.6$. ESI-HRMS $(m / z)$ : calcd. for $\left(\mathrm{C}_{18} \mathrm{H}_{15} \mathrm{~N}_{6} \mathrm{O}_{2}\right)[\mathrm{M}$ $+\mathrm{H}^{+}$] 347.1251, found 347.1254.

(2,4-Dimethylphenyl)-2H,8H-benzo[g]bis([1,2,3]triazolo)[5,1-c:4',5'-e][1,4]oxazocine (4m). Yield $253 \mathrm{mg}$ $(77 \%)$, white solid, m. p. $173^{\circ} \mathrm{C}$. IR $\left(v, \mathrm{~cm}^{-1}\right)$ : no signals of azido group and triple bond were observed. ${ }^{1} \mathrm{H}-\mathrm{NMR}: \delta 2.41(\mathrm{~s}, 3 \mathrm{H}), 2.52(\mathrm{~s}, 3 \mathrm{H}), 5.60(\mathrm{~s}, 2 \mathrm{H}), 7.16-7.29(\mathrm{~m}, 2 \mathrm{H}), 7.26-7.31(\mathrm{~m}, 2 \mathrm{H}), 7.46-7.50(\mathrm{~m}$, $1 \mathrm{H}), 7.65(\mathrm{t}, 2 \mathrm{H}, J=4.0 \mathrm{~Hz}), 7.70(\mathrm{dd}, 1 \mathrm{H}, J=7.6,1.6 \mathrm{~Hz}) .{ }^{13} \mathrm{C}-\mathrm{NMR}: \delta 19.0,21.1,67.0,121.5,121.9,125.0$, 125.5, 127.3, 130.1, 131.6, 131.6, 132.3, 132.4, 134.6, 136.8, 137.6, 139.4, 141.1, 155.7. ESI-HRMS (m/z): calcd. for $\left(\mathrm{C}_{19} \mathrm{H}_{17} \mathrm{~N}_{6} \mathrm{O}\right)\left[\mathrm{M}+\mathrm{H}^{+}\right] 345.1458$, found 345.1450.

(2,6-Dimethylphenyl)-2H,8H-benzo[g]bis([1,2,3]triazolo)[5,1-c:4',5'-e][1,4]oxazocine (4n). Yield $293 \mathrm{mg}$ $(85 \%)$, white solid, m. p. $175^{\circ} \mathrm{C}$. IR $\left(v, \mathrm{~cm}^{-1}\right)$ : no signals of azido group and triple bond were observed. ${ }^{1} \mathrm{H}-\mathrm{NMR}: \delta 2.21(\mathrm{~s}, 6 \mathrm{H}), 5.62(\mathrm{~s}, 2 \mathrm{H}), 7.21(\mathrm{~d}, 2 \mathrm{H}, J=7.6 \mathrm{~Hz}), 7.26-7.37(\mathrm{~m}, 3 \mathrm{H}), 7.46-7.50(\mathrm{~m}, 1 \mathrm{H}), 7.66$ (s, 1H), $7.72(\mathrm{dd}, 1 \mathrm{H}, J=7.7,1.6 \mathrm{~Hz}) .{ }^{13} \mathrm{C}-\mathrm{NMR}: \delta 17.6,67.2,121.6,121.8,125.5,128.4,130.0,130.2$, 131.6, 131.7, 134.5, 135.7, 137.4, 138.9, 141.1, 155.8. ESI-HRMS $(\mathrm{m} / \mathrm{z})$ : calcd. for $\left(\mathrm{C}_{19} \mathrm{H}_{17} \mathrm{~N}_{6} \mathrm{O}\right)\left[\mathrm{M}+\mathrm{H}^{+}\right]$ 345.1458 , found 345.1450 .

4-(2H,8H-Benzo[g]bis([1,2,3]triazolo)[5,1-c:4',5'-e][1,4]oxazocin-2-yl)benzonitrile (4o). Yield $178 \mathrm{mg}(52 \%)$, white solid, m. p. $198{ }^{\circ} \mathrm{C}$. IR $\left(v, \mathrm{~cm}^{-1}\right)$ : no signals of azido group and triple bond were observed. ${ }^{1} \mathrm{H}-\mathrm{NMR}: \delta 5.62(\mathrm{~s}, 2 \mathrm{H}), 7.31-7.35(\mathrm{~m}, 2 \mathrm{H}), 7.52-7.56(\mathrm{~m}, 1 \mathrm{H}), 7.65(\mathrm{~s}, 1 \mathrm{H}), 7.70(\mathrm{dd}, 1 \mathrm{H}, J=8.0,1.6 \mathrm{~Hz})$, $7.84(\mathrm{dt}, 2 \mathrm{H}, J=9.0,2.0 \mathrm{~Hz}), 8.34(\mathrm{dt}, 2 \mathrm{H}, J=9.0,2.0 \mathrm{~Hz}) .{ }^{13} \mathrm{C}-\mathrm{NMR}: \delta 67.4,111.7,115.5,118.0,119.1$, 121.4, 121.6, 125.8, 129.8, 131.6, 132.4, 133.6, 134.9, 139.9, 141.7, 142.9, 155.8. ESI-HRMS (m/z): calcd. for $\left(\mathrm{C}_{18} \mathrm{H}_{12} \mathrm{~N}_{7} \mathrm{O}\right)\left[\mathrm{M}+\mathrm{H}^{+}\right] 342.1098$, found 342.1096. 


\section{Conclusions}

The synthesis and photophysical properties of a series of new differently substituted 2-phenyl-2H,8H-benzo[g]bis([1,2,3]triazolo)[5,1-c:4',5'-e][1,4]oxazocines were investigated. The corresponding dichlorodiazenes containing propargyloxy groups were used as a key starting materials for this aim. Their reaction with sodium azide leads directly to the corresponding 4-azido-1,2,3-triazoles in up to 97\% yield. Subsequent thermal cyclization resulted in efficient synthesis of condensed heterocycles having an additional triazole ring in up to $86 \%$ yield. The prepared oxazocine derivatives demonstrated interesting photophysical properties and much higher fluorescence quantum yields in comparison to non-cyclized triazole derivatives.

Supplementary Materials: Copies of all the ${ }^{1} \mathrm{H}-$ and ${ }^{13} \mathrm{C}-\mathrm{NMR}$ spectra are available online.

Author Contributions: Conceptualization, V.N.; investigation, B.T.; writing-original draft preparation, B.T.; writing-review and editing, V.N.; visualization, B.T., V.N.; funding acquisition, V.N. All authors have read and agreed to the published version of the manuscript.

Funding: This research was funded by RFBR 18-53-06006 and 16-29-10669.

Acknowledgments: The authors acknowledge partial support in measuring of NMR and UV-Vis/Fluorescence Spectra from M.V. Lomonosov Moscow State University Program of Development. The authors acknowledge Thermo Fisher Scientific Inc., MS Analytica (Moscow, Russia).

Conflicts of Interest: The authors declare no conflict of interest.

\section{References}

1. Belskaya, N.; Subbotina, J.; Lesogorova, S. Synthesis of 2H-1,2,3-Triazoles. Top. Heterocycl. Chem. Ser. 2015, 40, 51-116.

2. Dehaen, W.; Bakulev, V.A. Chemistry of 1,2,3-triazoles. Top. Heterocycl. Chem. Ser. 2015, 40, 384. [CrossRef]

3. Krivolapov, V.P.; Shkurko, O.P. 1,2,3-Triazole and its derivatives. Development of methods for the formation of the triazole ring. Russ. Chem. Rev. 2005, 74, 339-379. [CrossRef]

4. Kalisiak, J.; Sharpless, K.B.; Fokin, V.V. Efficient synthesis of 2-substituted-1,2,3-triazoles. Org. Lett. 2008, 10, 3171-3174. [CrossRef] [PubMed]

5. Schulze, B.; Schubert, U.S. Beyond click chemistry-Supramolecular interactions of 1,2,3-triazoles. Chem. Soc. Rev. 2014, 43, 2522-2571. [CrossRef] [PubMed]

6. Huisgen, R. Centenary Lecture-1,3-Dipolar Cycloadditions. Proc. Chem. Soc. Lond. 1961, 357-396. [CrossRef]

7. Li, S.X.; Feng, L.R.; Guo, X.J.; Zhang, Q. Application of thermal azide-alkyne cycloaddition (TAAC) reaction as a low temperature cross-linking method in polymer gate dielectrics for organic field-effect transistors. J. Mater. Chem. C 2014, 2, 3517. [CrossRef]

8. Zhu, X.M.; Schmidt, R.R. Glycosylthiomethyl Chloride: A New Species for S-Neoglycoconjugate Synthesis. Synthesis of 1-N-Glycosylthiomethyl-1,2,3-triazoles. J. Org. Chem. 2004, 69, 1081-1085. [CrossRef]

9. Hager, C.; Mietchen, R.; Reinke,H. Organofluorine compounds and fluorinating agents. Part 26: New reversed nucleosides-perfluoroalkyl substituted 1,2,3-triazoles linked to D-galactose and D-altrose. J. Fluor. Chem. 2000, 104, 135. [CrossRef]

10. Delisi, R.; Mingoia, F.; Terenzi, A.; Martorana, A.; Barone, G.; Almerico, A. 1,2,3-Triazole in Heterocyclic Compounds, Endowed with Biological Activity, through 1,3-Dipolar Cycloadditions. Eur. J. Org. Chem. 2014, 2014, 3289-3306. [CrossRef]

11. Kosmrlj, J. Click triazoles. Top. Heterocycl. Chem. Ser. 2012, 28, 236. [CrossRef]

12. Kolb, H.C.; Finn, M.G.; Sharpless, K.B. Click Chemistry: Diverse Chemical Function from a Few Good Reactions. Angew. Chem. 2001, 40, 2004-2021. [CrossRef]

13. Alvares-Builla, J.; Vagquero, J.J.; Burluenga, J. Modern Heterocyclic Chemistry; Wiley VCH: Weinheim, Germany, 2011; Volume 12, pp. 989-1008.

14. Dheer, D.; Singh, V.; Shankar, R. Medicinal attributes of 1,2,3-triazoles: Current developments. Bioorg. Chem. 2017, 71, 30-54. [CrossRef] [PubMed] 
15. Kaur, R.; Dwivedi, A.R.; Kumar, B.; Kumar, V. Recent Developments on 1,2,4-Triazole Nucleus in Anticancer Compounds: A Review. Anticancer Agents Med. Chem. 2016, 16, 465-489. [CrossRef]

16. De Carvalho da Silva, F.; do Carmo Cardoso, M.F.; Ferreira, P.G.; Ferreira, V.F. Biological properties of 1Hand 2H-1,2,3-Triazoles. Top. Heterocycl. Chem. Ser. 2015, 40, 117-166.

17. Ma, N.; Wang, Y.; Zhao, B.X.; Ye, W.C.; Jiang, S. The application of click chemistry in the synthesis of agents with anticancer activity. Drug Des. Dev. Ther. 2015, 9, 1585-1599. [CrossRef]

18. Fletcher, R.A.; Gilley, A.; Sankhla, N.; Davis, T.D. Triazoles as Plant Growth Regulators and Stress Protectants. Hortic. Rev. 2010, 24, 55-138. [CrossRef]

19. Liu, Y.; Yan, W.; Chen, Y.; Petersen, J.L.; Shi, X. Efficient Synthesis of N-2-Aryl-1,2,3-Triazole Fluorophores via Post-Triazole Arylation. Org. Lett. 2008, 10, 5389-5392. [CrossRef]

20. Katan, C.; Savel, P.; Wong, B.M.; Roisnel, T.; Dorcet, V.; Jean-Luc Fillaut, J.L.; Jacquemin, D. Absorption and fluorescence signatures of 1,2,3-triazole based regioisomers: Challenging compounds for TD-DFT. Phys. Chem. Chem. Phys. 2014, 16, 9064-9073. [CrossRef]

21. Singh, H.; Sidhu, J.; Khurana, J.M. Synthesis and photophysical properties of novel chloroquinoline based chalcone derivates containing 1,2,3-triazole moiety. J. Lumin. 2015, 158, 340-350. [CrossRef]

22. Yan, W.; Wang, Q.; Lin, Q.; Li, M.; Petersen, J.L.; Shi, X. N-2-Aryl-1,2,3-triazoles: A Novel Class of UV/Blue-Light-Emitting Fluorophores with Tunable Optical Properties. Chem. Eur. J. 2011, 17, 5011-5018. [CrossRef] [PubMed]

23. Gavlik, K.D.; Sukhorukova, E.S.; Shafran, Y.M.; Slepukhin, P.A.; Benassi, E.; Belskaya, N.P. 2-Aryl-5-amino-1,2,3-triazoles: New effective blue-emitting fluorophores. Dyes Pigments 2017, 136, $229-242$. [CrossRef]

24. Padalkar, V.S.; Lanke, S.K.; Chemate, S.B.; Sekar, N. N-2-Aryl-1,2,3-Triazoles: A Novel Class of Blue Emitting Fluorophores-Synthesis, Photophysical Properties Study and DFT Computations. J. Lumin. 2015, 25, 985-996. [CrossRef]

25. Zhang, Y.; Ye, X.; Petersen, J.L.; Li, M.; Shi, X. Synthesis and characterization of Bis-N-2-Aryl Triazole as a fluorophore. J. Org. Chem. 2015, 80, 3664-3669. [CrossRef] [PubMed]

26. Nenajdenko, V.G.; Shastin, A.V.; Gorbachev, V.M.; Shorunov, S.V.; Muzalevskiy, V.M.; Lukianova, A.I.; Khrustalev, V.N. Copper-Catalyzed Transformation of Hydrazones into Halogenated Azabutadienes, Versatile Building Blocks for Organic Synthesis. ACS Catal. 2016, 7, 205-209. [CrossRef]

27. Shastin, A.V.; Sergeev, P.G.; Lukianova, A.I.; Muzalevskiy, V.M.; Khrustalev, V.N.; Dorovatovskii, P.V. Dichloro-Substituted 1,2-Diazabuta-1,3-dienes as Highly Reactive Electrophiles in the Reaction with Amines and Diamines: Efficient Synthesis of $\alpha$-Hydrazo Amidinium Salts. Eur. J. Org. Chem. 2018, 36, 4996-5006. [CrossRef]

28. Shikhaliyev, N.Q.; Ahmadova, N.E.; Gurbanov, A.V.; Maharramov, A.M.; Mammadova, G.Z.; Nenajdenko, V.G.; Zubkov, F.I.; Mahmudov, K.T.; Pombeiro, A.J.L. Noncovalent interactions in the design of bis-azo dyes. Dyes Pigments 2018, 150, 377-381. [CrossRef]

29. Maharramov, A.M.; Shikhaliyev, N.Q.; Suleymanova, G.T.; Gurbanov, A.V.; Babayeva, G.V.; Mammadova, G.Z.; Zubkov, F.I.; Nenajdenko, V.G.; Mahmudov, K.T.; Pombeiro, A.J.L. Pnicogen, halogen and hydrogen bonds in (E)-1-(2,2-dichloro-1-(2-nitrophenyl)vinyl)-2-(para-substituted phenyl)-diazenes. Dyes Pigments 2018, 159, 135-141. [CrossRef]

30. Shastin, A.V.; Tsyrenova, B.D.; Sergeev, P.G.; Roznyatovsky, V.A.; Smolyar, I.V.; Khrustalev, V.N.; Nenajdenko, V.G. Synthesis of a New Family of 1,1-Diazidoethenes: One-Pot Construction of 4-Azido-1,2,3-triazoles via Nitrene Cyclization. Org. Lett. 2018, 20, 7803-7806. [CrossRef]

31. Hans, R.H.; Guantai, E.M.; Lategan, C.; Smith, P.J.; Wan, B.; Franzblau, S.G.; Chibale, K. Synthesis, antimalarial and antitubercular activity of acetylenic chalcones. Bioorg. Med. Chem. Lett. 2010, 20, 942-944. [CrossRef]

32. Bräse, S.; Banert, K. Organic Azides: Syntheses and Applications; John Wiley \& Sons: Chichester, UK, 2010.

33. Sokolova, N.V.; Nenajdenko, V.G. Recent advances in the $\mathrm{Cu}(\mathrm{I})$-catalyzed azide-alkyne cycloaddition: Focus on functionally substituted azides and alkynes. RSC Adv. 2013, 3, 16212-16242. [CrossRef]

34. Ōki, M. Recent Advances in Atropisomerism. Top. Stereochem. Ser. 2007, 14, 1-81. [CrossRef]

35. Valeur, B. Molecular Fluorescence. Principles and Applications; Wiley VCH: Weinheim, Germany, 2002. [CrossRef]

36. Melhuish, W.H. Quantum efficiencies of fluorescence of organic substances: Effect of solvent and concentration of the fluorescent solute. J. Phys. Chem. 1961, 65, 229-235. [CrossRef] 
37. Muińo, P.L.; Callis, P.R. Solvent Effects on the Fluorescence Quenching of Tryptophan by Amides via Electron Transfer. Experimental and Computational Studies. J. Phys. Chem. 2009, 113, 2572-2577. [CrossRef]

Sample Availability: Samples of the compounds are available from the authors. article distributed under the terms and conditions of the Creative Commons Attribution (CC BY) license (http://creativecommons.org/licenses/by/4.0/). 